\title{
A degenerate elliptic-parabolic problem with nonlinear dynamical boundary conditions
}

\author{
F. ANDREU† J. M. MAZÓN\$ J. TOLEDO ${ }^{\S}$ \\ Departamento de Análisis Matemático, Universitat de València, \\ Dr. Moliner 50, 46100 Burjassot (Valencia), Spain \\ N. IGBIDA ${ }^{\mathrm{II}}$ \\ LAMFA, CNRS-UMR 6140, Université de Picardie Jules Verne, \\ 33 rue Saint Leu, 80038 Amiens, France
}

[Received 22 November 2005 and in revised form 12 May 2006]

\begin{abstract}
We prove existence and uniqueness of weak solutions for a general degenerate elliptic-parabolic problem with nonlinear dynamical boundary conditions. Particular instances of this problem appear in various phenomena with changes of phase like the multiphase Stefan problem and in the weak formulation of the mathematical model of the so called Hele-Shaw problem. Also, the problem with nonhomogeneous Neumann boundary conditions is included.
\end{abstract}

2000 Mathematics Subject Classification: 35J60, 35D02.

\section{Introduction}

The purpose of this paper is to establish the existence and uniqueness of a weak solution for a degenerate elliptic-parabolic problem with nonlinear dynamical boundary condition of the form

$$
P_{\gamma, \beta}\left(f, g, z_{0}, w_{0}\right) \quad\left\{\begin{array}{l}
\left.z_{t}-\operatorname{div} \mathbf{a}(x, D u)=f, \quad z \in \gamma(u), \quad \text { in } Q_{T}:=\right] 0, T[\times \Omega, \\
\left.w_{t}+\mathbf{a}(x, D u) \cdot \eta=g, \quad w \in \beta(u), \quad \text { on } S_{T}:=\right] 0, T[\times \partial \Omega, \\
z(0)=z_{0} \quad \text { in } \Omega, \quad w(0)=w_{0} \quad \text { in } \partial \Omega,
\end{array}\right.
$$

where $T>0, \Omega$ is a bounded domain in $\mathbb{R}^{N}$ with smooth boundary $\partial \Omega, z_{0} \in L^{1}(\Omega), w_{0} \in$ $L^{1}(\partial \Omega), f \in L^{1}\left(0, T ; L^{1}(\Omega)\right), g \in L^{1}\left(0, T ; L^{1}(\partial \Omega)\right)$ and $\eta$ is the unit outward normal on $\partial \Omega$. Here a : $\Omega \times \mathbb{R}^{N} \rightarrow \mathbb{R}^{N}$ is a Carathéodory function satisfying the classical Leray-Lions conditions. The nonlinearities $\gamma$ and $\beta$ are maximal monotone graphs in $\mathbb{R}^{2}$ (see, e.g., [21]) such that $0 \in \gamma(0)$, $\operatorname{Dom}(\gamma)=\mathbb{R}$, and $0 \in \beta(0)$. In particular, $\gamma$ and $\beta$ may be multivalued. This allows one to include the homogeneous Dirichlet boundary condition (taking $\beta$ to be the monotone graph $D=\{0\} \times \mathbb{R}$ ), in which case we consider, in fact, the following problem with static boundary conditions:

$$
D P_{\gamma}\left(f, z_{0}\right) \quad\left\{\begin{array}{l}
z_{t}-\operatorname{div} \mathbf{a}(x, D u)=f, \quad z \in \gamma(u), \quad \text { in } Q_{T}, \\
u=0 \quad \text { on } S_{T}, \\
z(0)=z_{0} \quad \text { in } \Omega
\end{array}\right.
$$

\footnotetext{
${ }^{\dagger}$ E-mail: Fuensanta.Andreu@uv.es

‡E-mail: mazon@uv.es

E-mail: toledojj@uv.es

${ }^{\mathbb{I}}$ E-mail: noureddine.igbida@u-picardie.fr
} 
and the nonhomogeneous Neumann boundary condition (taking $\beta$ to be the monotone graph $\mathrm{N}$ defined by $\mathrm{N}(r)=0$ for all $r \in \mathbb{R})$, in which case we consider the problem

$$
N P_{\gamma}\left(f, g, z_{0}\right) \quad\left\{\begin{array}{l}
z_{t}-\operatorname{div} \mathbf{a}(x, D u)=f, \quad z \in \gamma(u), \quad \text { in } Q_{T}, \\
\mathbf{a}(x, D u) \cdot \eta=g \quad \text { on } S_{T}, \\
z(0)=z_{0} \quad \text { in } \Omega
\end{array}\right.
$$

as well as many other nonlinear fluxes on the boundary that occur in mechanics and physics (see, e.g., [28] or [20]). Note also that, since $\gamma$ may be multivalued, problems of type $P_{\gamma, \beta}\left(f, g, z_{0}, w_{0}\right)$ appear in various phenomena with changes of phase like the multiphase Stefan problem ([26]) and in the weak formulation of the mathematical model of the so called Hele-Shaw problem (see [27] and [29]). Moreover, if $\gamma=\mathrm{N}$, we consider the following elliptic problem with nonlinear dynamical boundary conditions:

$$
B P_{\beta}\left(f, g, w_{0}\right) \quad\left\{\begin{array}{l}
-\operatorname{div} \mathbf{a}(x, D u)=f \quad \text { in } Q_{T}, \\
w_{t}+\mathbf{a}(x, D u) \cdot \eta=g, \quad w \in \beta(u), \quad \text { on } S_{T}, \\
w(0)=w_{0} \quad \text { in } \partial \Omega .
\end{array}\right.
$$

The dynamical boundary conditions, although not too widely considered in the mathematical literature, are very natural in many mathematical models including heat transfer in a solid in contact with a moving fluid, thermoelasticity, diffusion phenomena, problems in fluid dynamics, etc. (see [9], [24], [30], [48] and the references therein). These dynamical boundary conditions also appear in the study of the Stefan problem when the boundary material has large thermal conductivity and sufficiently small thickness. Hence, the boundary material is regarded as the boundary of the domain. For instance, this is the case if one considers an iron ball in which water and ice coexist. For more details about these physical considerations one can see for instance [1]. They also appear in the study of the Hele-Shaw problem. Recall that in [27] the authors give the weak formulation of the problem in the form of a nonlinear degenerate parabolic problem, governed by the Laplace operator and the multivalued Heaviside function, with static boundary conditions. From the physical point of view they assume that the prescribed value of the flux on the boundary is known. But, in some practical situations, it may not be possible to prescribe or to control the exact value of the flux on the boundary. In [47] (see also [49]), the authors consider the case of nonlocal dynamical boundary conditions and use variational methods to solve the problem.

In the present paper, we cover the case of general nonlinear diffusion and local dynamical boundary conditions. Notice that general nonlinear diffusion operators of Leray-Lions type, different from the Laplacian, appear when one deals with non-Newtonian fluids (see, e.g., [8], [42], [43] and the references therein for the case of the Hele-Shaw problem with non-Newtonian fluids). Another interesting application we have in mind concerns the filtration equation with dynamical boundary conditions (see, e.g., [50]), which appears for example in the study of rainfall infiltration through soil, when accumulation of water on the ground surfaces caused by saturation of the surface layer is taken into account. Observe that $\beta$ may be such that $\operatorname{Ran}(\beta)$ is different from $\mathbb{R}$, so that we cover the case where the boundary conditions are either dynamical or static with respect to the values of $w$ in the problem under consideration. This is the situation where the saturation happens only for values of $w$ in a subinterval of $\mathbb{R}$.

In contrast to the case of Dirichlet boundary conditions (problem $D P_{\gamma}\left(f, z_{0}\right)$ ), which is well known (see [2], [4], [16], [18], [22], [39] and the references therein), to our knowledge there is little 
literature on problems $P_{\gamma, \beta}\left(f, g, z_{0}, w_{0}\right)$, and the results mostly concern particular nonlinearities and the Laplace operator. For instance, the problem $N P_{\gamma}\left(f, g, z_{0}\right)$ is treated by Hulshof in [33] in the particular case where $\gamma$ is a uniformly Lipschitz continuous function, $\gamma(r)=1$ for $r \in \mathbb{R}^{+}, \gamma \in$ $C^{1}\left(\mathbb{R}^{-}\right), \gamma^{\prime}>0$ on $\mathbb{R}^{-}$and $\lim _{r \downarrow-\infty} \gamma(r)=0$ and for some particular functions $g$. Kenmochi in [40] considers the same problem in the case $\gamma \in C(\mathbb{R})$ with $\operatorname{Ran}(\gamma)$ a closed bounded interval. The second author of the present paper studies the cases where $\gamma$ is the Heaviside maximal monotone graph and the case where $\gamma(r)=\exp (r)$ in [34] and [36], respectively. In one space dimension, much more literature exists (see [17] and [51] and the references therein).

For elliptic-parabolic problems with dynamical boundary conditions, the cases in which $\gamma$ and $\beta$ are both linear are well known (see, e.g., [32], [30], [31], [44], [3] and the references therein). For the general nonlinear case, that is, when $\gamma$ and $\beta$ are maximal monotone graphs, most of the papers in the literature concern the Laplace operator and $\gamma$ and $\beta$ with range $\mathbb{R}$ (see [50], [1] and the references therein). The problem becomes more complicated if one of the ranges of $\gamma$ and $\beta$ may not be equal to $\mathbb{R}$, and there are few relevant results in the literature. In [35] the case where $\beta$ is a continuous nondecreasing function (possibly depending on $x$ ) and $\gamma$ is the Heaviside maximal monotone graph, which corresponds to the Hele-Shaw problem, is studied. In [38], the authors consider the homogeneous case, i.e., $f=0$ and $g=0$, with $\gamma$ and $\beta$ being maximal monotone graphs everywhere defined.

Roughly speaking, in contrast to the Dirichlet boundary condition, for the nonhomogeneous Neumann boundary condition and/or dynamical boundary conditions, the problem is noncoercive, and moreover, the conservation of mass exhibits a necessary condition for the existence of a solution related to the ranges of the nonlinearities $\gamma$ and $\beta$ (see (6)). Indeed, prescribing the value of $u$ on some part of the lateral boundary, one can control the Sobolev norm of the solution in the interior of $\Omega$ by the $L^{p}$ norm of the gradient in $\Omega$. This is not possible in the case of purely Neumann boundary conditions or dynamical ones, and some substitute for this kind of argument has to be found. In the case where the nonlinearities have ranges equal to $\mathbb{R}$ and assuming additional assumptions on $f$ and $g$ one can obtain $L^{\infty}$-estimates for the solutions (see, e.g., [33] and [38]). If one of the ranges is not equal to $\mathbb{R}$, the $L^{\infty}$-estimates are lost and the existence of solutions becomes complicated.

Another main difficulty when dealing with doubly nonlinear parabolic problems is the uniqueness. For the Laplace operator, thanks to the linearity of the operator, the problem can be solved by using suitable test functions with respect to $u$ (see, e.g., [38]). For nonlinear operators this kind of argument cannot be used. In [16], for an elliptic-parabolic problem with Dirichlet boundary conditions, it is shown that the notion of integral solution ([10]) is a very useful tool to prove uniqueness (see also [37] for nonhomogeneous and time dependent Neumann boundary conditions). For general nonlinearities, even for homogeneous Dirichlet boundary condition, the question of uniqueness is more difficult and most of the arguments used in the literature are based on doubling variable methods (see, e.g., [22], [23], [39], [18], [5] and the references therein). In this paper we show that applying the notion of integral solution simplifies the proof of uniqueness, which is obtained without using doubling variable methods. Moreover with this technique uniqueness is proved without any assumption on the jumps of $\gamma$ and $\beta$.

We also make use of nonlinear semigroup theory ([14], [52]). So we need to consider the elliptic problem

$$
\left(S_{\phi, \psi}^{\gamma, \beta}\right) \quad \begin{cases}-\operatorname{div} \mathbf{a}(x, D u)+\gamma(u) \ni \phi & \text { in } \Omega, \\ \mathbf{a}(x, D u) \cdot \eta+\beta(u) \ni \psi & \text { on } \partial \Omega .\end{cases}
$$


In [6], under rather general assumptions, existence of solutions and a contraction principle for problem $\left(S_{\phi, \psi}^{\gamma, \beta}\right)$ are obtained. Using these results we prove the existence of mild solutions for the associated Cauchy problem. In principle it is not clear how these mild solutions have to be interpreted for problem $P_{\gamma, \beta}\left(f, g, z_{0}, w_{0}\right)$. Under some additional natural conditions (see Theorem 3.3. we show that mild solutions are weak solutions of problem $P_{\gamma, \beta}\left(f, g, z_{0}, w_{0}\right)$. To get uniqueness, we show that weak solutions are integral solutions.

Let us briefly summarize the content of the paper. In Section 2 we fix the notation and give some preliminaries; we also introduce the concept of weak solution for problem $P_{\gamma, \beta}\left(f, g, z_{0}, w_{0}\right)$ and state the existence and uniqueness result for weak solutions of $P_{\gamma, \beta}\left(f, g, z_{0}, w_{0}\right)$ and a contraction principle satisfied by weak solutions. In Section 3 we study the problem from the point of view of nonlinear semigroup theory. In Section 4 we prove the existence of weak solutions and in Section 5 we obtain a contraction principle. Finally, in the appendix we give the proof of the characterization of the closure of the domain of the operator associated to our problem.

\section{Preliminaries and main result}

In this section, after some preliminaries, we give the concept of weak solution for problem $P_{\gamma, \beta}\left(f, g, z_{0}, w_{0}\right)$ and we state the existence and uniqueness result for this type of solution.

Throughout the paper, $\Omega \subset \mathbb{R}$ is a bounded domain with smooth boundary $\partial \Omega, p>1, \gamma$ and $\beta$ are maximal monotone graphs in $\mathbb{R}^{2}$ such that $\operatorname{Dom}(\gamma)=\mathbb{R}, 0 \in \gamma(0) \cap \beta(0)$, and the Carathéodory function $\mathbf{a}: \Omega \times \mathbb{R}^{N} \rightarrow \mathbb{R}^{N}$ satisfies

$\left(\mathrm{H}_{1}\right)$ there exists $\lambda>0$ such that $\mathbf{a}(x, \xi) \cdot \xi \geqslant \lambda|\xi|^{p}$ for a.e. $x \in \Omega$ and for all $\xi \in \mathbb{R}^{N}$,

$\left(\mathrm{H}_{2}\right)$ there exist $c>0$ and $\varrho \in L^{p^{\prime}}(\Omega)$ such that $|\mathbf{a}(x, \xi)| \leqslant \sigma\left(\varrho(x)+|\xi|^{p-1}\right)$ for a.e. $x \in \Omega$ and for all $\xi \in \mathbb{R}^{N}$, where $p^{\prime}=p /(p-1)$,

( $\left.\mathrm{H}_{3}\right)(\mathbf{a}(x, \xi)-\mathbf{a}(x, \eta)) \cdot(\xi-\eta)>0$ for a.e. $x \in \Omega$ and for all $\xi, \eta \in \mathbb{R}^{N}, \xi \neq \eta$.

The hypotheses $\left(\mathrm{H}_{1}\right)-\left(\mathrm{H}_{3}\right)$ are classical in the study of nonlinear operators in divergence form (see, e.g., [46] or [11]). The model example of a function a satisfying these hypotheses is $\mathbf{a}(x, \xi)=$ $|\xi|^{p-2} \xi$. The corresponding operator is the $p$-Laplacian operator $\Delta_{p}(u)=\operatorname{div}\left(|D u|^{p-2} D u\right)$.

We denote by $|A|$ the Lebesgue measure of a set $A \subset \mathbb{R}^{N}$ or its $(N-1)$-Hausdorff measure. For $1 \leqslant q<+\infty, L^{q}(\Omega)$ and $W^{1, q}(\Omega)$ denote respectively the standard Lebesgue and Sobolev spaces, and $W_{0}^{1, q}(\Omega)$ is the closure of $\mathcal{D}(\Omega)$ in $W^{1, q}(\Omega)$. For $u \in W^{1, q}(\Omega)$, we denote by $u$ or $\operatorname{tr}(u)$ the trace of $u$ on $\partial \Omega$ in the usual sense. Recall that $\operatorname{tr}\left(W^{1, q}(\Omega)\right)=W^{1 / q^{\prime}, q}(\partial \Omega)$ and $\operatorname{Ker}(\operatorname{tr})=W_{0}^{1, q}(\Omega)$.

We introduce the sets

$$
V^{1, q}(\Omega):=\left\{\phi \in L^{1}(\Omega): \exists M>0 \text { such that } \int_{\Omega}|\phi v| \leqslant M\|v\|_{W^{1, q}(\Omega)} \forall v \in W^{1, q}(\Omega)\right\}
$$

and

$$
V^{1, q}(\partial \Omega):=\left\{\psi \in L^{1}(\partial \Omega): \exists M>0 \text { such that } \int_{\partial \Omega}|\psi v| \leqslant M\|v\|_{W^{1, q}(\Omega)} \forall v \in W^{1, q}(\Omega)\right\} .
$$

$V^{1, q}(\Omega)$ is a Banach space endowed with the norm

$$
\|\phi\|_{V^{1, q}(\Omega)}:=\inf \left\{M>0: \int_{\Omega}|\phi v| \leqslant M\|v\|_{W^{1, q}(\Omega)} \forall v \in W^{1, q}(\Omega)\right\},
$$


and $V^{1, q}(\partial \Omega)$ is a Banach space endowed with the norm

$$
\|\psi\|_{V^{1, q}(\partial \Omega)}:=\inf \left\{M>0: \int_{\partial \Omega}|\psi v| \leqslant M\|v\|_{W^{1, q}(\Omega)} \forall v \in W^{1, q}(\Omega)\right\} .
$$

Observe that Sobolev embeddings and trace theorems imply, for $1 \leqslant q<N$,

$$
L^{q^{\prime}}(\Omega) \subset L^{(N q /(N-q))^{\prime}}(\Omega) \subset V^{1, q}(\Omega)
$$

and

$$
L^{q^{\prime}}(\partial \Omega) \subset L^{((N-1) q /(N-q))^{\prime}}(\partial \Omega) \subset V^{1, q}(\partial \Omega) .
$$

Also,

$$
\begin{aligned}
& V^{1, q}(\Omega)=L^{1}(\Omega) \quad \text { and } \quad V^{1, q}(\partial \Omega)=L^{1}(\partial \Omega) \quad \text { when } q>N \text {, } \\
& L^{q}(\Omega) \subset V^{1, N}(\Omega) \quad \text { and } \quad L^{q}(\partial \Omega) \subset V^{1, N}(\partial \Omega) \quad \text { for any } q>1 \text {. }
\end{aligned}
$$

We say that a is smooth (see [7] and [6]) when, for any $\phi \in L^{\infty}(\Omega)$ such that there exists a bounded weak solution $u$ of the homogeneous Dirichlet problem

$$
\text { (D) } \begin{cases}-\operatorname{div} \mathbf{a}(x, D u)=\phi & \text { in } \Omega, \\ u=0 & \text { on } \partial \Omega\end{cases}
$$

there exists $\psi \in L^{1}(\partial \Omega)$ such that $u$ is also a weak solution of the Neumann problem

$$
\text { (N) } \begin{cases}-\operatorname{div} \mathbf{a}(x, D u)=\phi & \text { in } \Omega, \\ \mathbf{a}(x, D u) \cdot \eta=\psi & \text { on } \partial \Omega .\end{cases}
$$

Functions a corresponding to linear operators with smooth coefficients and $p$-Laplacian type operators are smooth (see [20] and [45]). In [6], we prove that a is smooth if and only if for any $\phi \in V^{1, p}(\Omega)$ there exists $\psi=T(\phi) \in V^{1, p}(\partial \Omega)$ such that the weak solution $u$ of $(D)$ is a weak solution of $(N)$. Moreover,

$$
\int_{\Omega}\left(T\left(\phi_{1}\right)-T\left(\phi_{2}\right)\right)^{+} \leqslant \int_{\Omega}\left(\phi_{1}-\phi_{2}\right)^{+} \quad \text { for all } \phi_{1}, \phi_{2} \in V^{1, p}(\Omega) .
$$

For a maximal monotone graph $\vartheta$ in $\mathbb{R} \times \mathbb{R}$ the main section $\vartheta^{0}$ of $\vartheta$ is defined by

$$
\vartheta^{0}(s):= \begin{cases}\text { the element of minimal absolute value of } \vartheta(s) \quad \text { if } \vartheta(s) \neq \emptyset, \\ +\infty & \text { if }[s,+\infty) \cap \operatorname{Dom}(\vartheta)=\emptyset, \\ -\infty & \text { if }(-\infty, s] \cap \operatorname{Dom}(\vartheta)=\emptyset .\end{cases}
$$

We write $\vartheta_{-}:=\inf \operatorname{Ran}(\vartheta)$ and $\vartheta_{+}:=\sup \operatorname{Ran}(\vartheta)$. If $0 \in \operatorname{Dom}(\vartheta)$, then $j_{\vartheta}(r)=\int_{0}^{r} \vartheta^{0}(s) \mathrm{d} s$ defines a convex 1.s.c. function such that $\vartheta=\partial j_{\vartheta}$. If $j_{\vartheta}^{*}$ is the Legendre transform of $j_{\vartheta}$ then $\vartheta^{-1}=\partial j_{\vartheta}^{*}$.

In [13] the following relation for $u, v \in L^{1}(\Omega)$ is defined: $u \ll v$ if

$$
\int_{\Omega}(u-k)^{+} \leqslant \int_{\Omega}(v-k)^{+} \quad \text { and } \quad \int_{\Omega}(u+k)^{-} \leqslant \int_{\Omega}(v+k)^{-} \quad \text { for any } k>0,
$$

and the following facts are proved. 
Proposition 2.1 Let $\Omega$ be a bounded domain in $\mathbb{R}^{N}$.

(i) If $u, v \in L^{1}(\Omega)$ and $u \ll v$, then $\|u\|_{q} \leqslant\|v\|_{q}$ for any $q \in[1,+\infty]$.

(ii) If $v \in L^{1}(\Omega)$, then $\left\{u \in L^{1}(\Omega): u \ll v\right\}$ is a weakly compact subset of $L^{1}(\Omega)$.

As said in the introduction, our aim is to study the existence and uniqueness of a weak solution for problem $P_{\gamma, \beta}\left(f, g, z_{0}, w_{0}\right)$. The concept of weak solution we have in mind is the following.

Definition 2.2 Given $f \in L^{1}\left(0, T ; L^{1}(\Omega)\right), g \in L^{1}\left(0, T ; L^{1}(\partial \Omega)\right), z_{0} \in L^{1}(\Omega)$ and $w_{0} \in L^{1}(\partial \Omega)$, a weak solution of $P_{\gamma, \beta}\left(f, g, z_{0}, w_{0}\right)$ in $[0, T]$ is a couple $(z, w)$ such that $z \in C\left([0, T] ; L^{1}(\Omega)\right), w \in C\left([0, T] ; L^{1}(\partial \Omega)\right), z(0)=z_{0}, w(0)=w_{0}$ and there exists $u \in L^{p}\left(0, T ; W^{1, p}(\Omega)\right)$ such that $z \in \gamma(u)$ a.e. in $Q_{T}, w \in \beta(u)$ a.e. on $S_{T}$ and

$$
\frac{\mathrm{d}}{\mathrm{d} t} \int_{\Omega} z(t) \xi+\frac{\mathrm{d}}{\mathrm{d} t} \int_{\partial \Omega} w(t) \xi+\int_{\Omega} \mathbf{a}(x, D u(t)) \cdot D \xi=\int_{\Omega} f(t) \xi+\int_{\partial \Omega} g(t) \xi \quad \text { in } \mathcal{D}^{\prime}(] 0, T[)
$$

for any $\xi \in C^{1}(\bar{\Omega})$.

REMARK 2.3 Observe that taking $\xi=1$ in the above definition, we get

$$
\int_{\Omega} z(t)+\int_{\partial \Omega} w(t)=\int_{\Omega} z_{0}+\int_{\partial \Omega} w_{0}+\int_{0}^{t}\left(\int_{\Omega} f+\int_{\partial \Omega} g\right) \quad \forall t \in[0, T] .
$$

Recall that in the case $\beta=0$, for the Laplacian operator and $\gamma$ the multivalued Heaviside function (i.e., for the Hele-Shaw problem), existence and uniqueness of weak solutions for this problem is known to hold only if

$$
\left.\int_{\Omega} z_{0}+\int_{0}^{t}\left(\int_{\Omega} f+\int_{\partial \Omega} g\right) \in\right] 0,|\Omega|[\quad \text { for any } t \in[0, T[
$$

(see [34] or [40])). For the maximal monotone graphs $\gamma$ and $\beta$, we set

$$
\mathcal{R}_{\gamma, \beta}^{+}:=\gamma_{+}|\Omega|+\beta_{+}|\partial \Omega|, \quad \mathcal{R}_{\gamma, \beta}^{-}:=\gamma_{-}|\Omega|+\beta_{-}|\partial \Omega|
$$

We suppose $\mathcal{R}_{\gamma, \beta}^{-}<\mathcal{R}_{\gamma, \beta}^{+}$and we write $\left.\mathcal{R}_{\gamma, \beta}:=\right] \mathcal{R}_{\gamma, \beta}^{-}, \mathcal{R}_{\gamma, \beta}^{+}[$.

The main results of this paper are the following contraction principle and the following existence and uniqueness theorem.

THEOREM 2.4 Let $T>0$. For $i=1$, 2, let $f_{i} \in L^{1}\left(0, T ; L^{1}(\Omega)\right), g_{i} \in L^{1}\left(0, T ; L^{1}(\partial \Omega)\right)$, $z_{i 0} \in L^{1}(\Omega)$ and $w_{i 0} \in L^{1}(\partial \Omega)$; let $\left(z_{i}, w_{i}\right)$ be a weak solution in $[0, T]$ of problem $P_{\gamma, \beta}\left(f_{i}, g_{i}, z_{i 0}, w_{i 0}\right), i=1,2$. Then

$$
\begin{aligned}
\int_{\Omega}\left(z_{1}(t)-z_{2}(t)\right)^{+}+\int_{\partial \Omega} & \left(w_{1}(t)-w_{2}(t)\right)^{+} \leqslant \int_{\Omega}\left(z_{10}-z_{20}\right)^{+}+\int_{\partial \Omega}\left(w_{10}-w_{20}\right)^{+} \\
& +\int_{0}^{t} \int_{\Omega}\left(f_{1}(\tau)-f_{2}(\tau)\right)^{+} \mathrm{d} \tau+\int_{0}^{t} \int_{\partial \Omega}\left(g_{1}(\tau)-g_{2}(\tau)\right)^{+} \mathrm{d} \tau
\end{aligned}
$$

for almost every $t \in] 0, T[$. 
TheORem 2.5 Assume $\operatorname{Dom}(\gamma)=\mathbb{R}, \mathcal{R}_{\gamma, \beta}^{-}<\mathcal{R}_{\gamma, \beta}^{+}$, and $\operatorname{Dom}(\beta)=\mathbb{R}$ or a smooth. Let $T>0$. Let $f \in L^{p^{\prime}}\left(0, T ; L^{p^{\prime}}(\Omega)\right), g \in L^{p^{\prime}}\left(0, T ; L^{p^{\prime}}(\partial \Omega)\right), z_{0} \in L^{p^{\prime}}(\Omega)$ and $w_{0} \in L^{p^{\prime}}(\partial \Omega)$ be such that

$$
\begin{gathered}
\gamma_{-} \leqslant z_{0} \leqslant \gamma_{+}, \quad \beta_{-} \leqslant w_{0} \leqslant \beta_{+}, \\
\int_{\Omega} j_{\gamma}^{*}\left(z_{0}\right)+\int_{\Gamma} j_{\beta}^{*}\left(w_{0}\right)<+\infty
\end{gathered}
$$

and

$$
\int_{\Omega} z_{0}+\int_{\partial \Omega} w_{0}+\int_{0}^{t}\left(\int_{\Omega} f+\int_{\partial \Omega} g\right) \in \mathcal{R}_{\gamma, \beta} \quad \forall t \in[0, T] .
$$

Then there exists a unique weak solution $(z, w)$ of problem $P_{\gamma, \beta}\left(f, g, z_{0}, w_{0}\right)$ in $[0, T]$.

The uniqueness part of Theorem 2.5 follows from Theorem 2.4 To prove Theorem 2.4 and the existence part of Theorem 2.5 we shall use the theory of nonlinear semigroups (see, e.g., [10], [14] or [25]). We will show the existence of a mild solution and we will prove that it is a weak solution of problem $P_{\gamma, \beta}\left(f, g, z_{0}, w_{0}\right)$. To prove the contraction principle we will show that weak solutions are integral solutions. To do this we need to rewrite $P_{\gamma, \beta}\left(f, g, z_{0}, w_{0}\right)$ as an abstract Cauchy problem and to use the results obtained in [6] for the associated elliptic problem.

\section{Mild solutions}

First let us recall some basic facts for the elliptic problem $\left(S_{\phi, \psi}^{\gamma, \beta}\right)$ given in [6], which will be crucial for the proof of our main results. In [6] the following concept of solution for problem $\left(S_{\phi, \psi}^{\gamma, \beta}\right)$ is introduced.

Definition 3.1 Let $\phi \in L^{1}(\Omega)$ and $\psi \in L^{1}(\partial \Omega)$. A triple of functions $[u, z, w] \in W^{1, p}(\Omega) \times$ $L^{1}(\Omega) \times L^{1}(\partial \Omega)$ is a weak solution of problem $\left(S_{\phi, \psi}^{\gamma, \beta}\right)$ if $z(x) \in \gamma(u(x))$ a.e. in $\Omega, w(x) \in \beta(u(x))$ a.e. in $\partial \Omega$, and

$$
\int_{\Omega} \mathbf{a}(x, D u) \cdot D v+\int_{\Omega} z v+\int_{\partial \Omega} w v=\int_{\partial \Omega} \psi v+\int_{\Omega} \phi v
$$

for all $v \in L^{\infty}(\Omega) \cap W^{1, p}(\Omega)$.

Observe that if $\left(S_{\phi, \psi}^{\gamma, \beta}\right)$ has a weak solution, then necessarily $\phi$ and $\psi$ must satisfy

$$
\mathcal{R}_{\gamma, \beta}^{-} \leqslant \int_{\partial \Omega} \psi+\int_{\Omega} \phi \leqslant \mathcal{R}_{\gamma, \beta}^{+} .
$$

Indeed, by taking $v=1$ as a test function in (7), we get

$$
\int_{\Omega} z+\int_{\partial \Omega} w=\int_{\partial \Omega} \psi+\int_{\Omega} \phi
$$

Moreover the following existence and uniqueness results about weak solutions of problem $\left(S_{\phi, \psi}^{\gamma, \beta}\right)$ have been obtained in [6]. 
THEOREM 3.2 (i) Let $\phi \in L^{1}(\Omega)$ and $\psi \in L^{1}(\partial \Omega)$, and let $\left[u_{1}, z_{1}, w_{1}\right]$ and $\left[u_{2}, z_{2}, w_{2}\right]$ be weak solutions of problem $\left(S_{\phi, \psi}^{\gamma, \beta}\right)$. Then there exists a constant $c \in \mathbb{R}$ such that

$$
\begin{aligned}
u_{1}-u_{2}=c & \text { a.e. in } \Omega, \\
z_{1}-z_{2}=0 & \text { a.e. in } \Omega, \\
w_{1}-w_{2}=0 & \text { a.e. in } \partial \Omega .
\end{aligned}
$$

Moreover, if $c \neq 0$, there exists a constant $k \in \mathbb{R}$ such that $z_{1}=z_{2}=k$.

(ii) For any weak solution $\left[u_{1}, z_{1}, w_{1}\right]$ of problem $\left(S_{\phi_{1}, \psi_{1}}^{\gamma, \beta}\right), \phi_{1} \in L^{1}(\Omega), \psi_{1} \in L^{1}(\partial \Omega)$, and any weak solution $\left[u_{2}, z_{2}, w_{2}\right]$ of problem $\left(S_{\phi_{2}, \psi_{2}}^{\gamma, \beta}\right), \phi_{2} \in L^{1}(\Omega), \psi_{2} \in L^{1}(\partial \Omega)$, we have

$$
\int_{\Omega}\left(z_{1}-z_{2}\right)^{+}+\int_{\partial \Omega}\left(w_{1}-w_{2}\right)^{+} \leqslant \int_{\partial \Omega}\left(\psi_{1}-\psi_{2}\right)^{+}+\int_{\Omega}\left(\phi_{1}-\phi_{2}\right)^{+} .
$$

Theorem 3.2 (ii) is given in [6] in a different way. With the technique used in Section 5 we can get exactly the above result.

Theorem 3.3 Assume $\operatorname{Dom}(\gamma)=\mathbb{R}$, and $\operatorname{Dom}(\beta)=\mathbb{R}$ or a smooth. For any $\phi \in V^{1, p}(\Omega)$ and $\psi \in V^{1, p}(\partial \Omega)$ with

$$
\int_{\Omega} \phi+\int_{\partial \Omega} \psi \in \mathcal{R}_{\gamma, \beta}
$$

there exists a weak solution $[u, z, w]$ of problem $\left(S_{\phi, \psi}^{\gamma, \beta}\right)$. Moreover $z \in V^{1, p}(\Omega), w \in V^{1, p}(\partial \Omega)$ and

$$
\int_{\Omega} \mathbf{a}(x, D u) \cdot D v+\int_{\Omega} z v+\int_{\partial \Omega} w v=\int_{\partial \Omega} \psi v+\int_{\Omega} \phi v \quad \text { for any } v \in W^{1, p}(\Omega) .
$$

These results imply that the natural space to study problem $P_{\gamma, \beta}\left(f, g, z_{0}, w_{0}\right)$ from the point of view of nonlinear semigroup theory is $X=L^{1}(\Omega) \times L^{1}(\partial \Omega)$ provided with the natural norm

$$
\|(f, g)\|:=\|f\|_{L^{1}(\Omega)}+\|g\|_{L^{1}(\partial \Omega)} .
$$

In this space we define the following operator

$$
\begin{aligned}
\mathcal{B}^{\gamma, \beta}:=\left\{((z, w),(\hat{z}, \hat{w})) \in X \times X: \exists u \in W^{1, p}(\Omega)\right. \text { such that } \\
\left.\quad[u, z, w] \text { is a weak solution of }\left(S_{z+\hat{z}, w+\hat{w}}^{\gamma, \beta}\right)\right\},
\end{aligned}
$$

in other words, $(\hat{z}, \hat{w}) \in \mathcal{B}^{\gamma, \beta}(z, w)$ if and only if there exists $u \in W^{1, p}(\Omega)$ such that $z(x) \in$ $\gamma(u(x))$ a.e. in $\Omega, w(x) \in \beta(u(x))$ a.e. in $\partial \Omega$, and

$$
\int_{\Omega} \mathbf{a}(x, D u) \cdot D v=\int_{\Omega} \hat{z} v+\int_{\partial \Omega} \hat{w} v
$$

for all $v \in L^{\infty}(\Omega) \cap W^{1, p}(\Omega)$, which allows us to rewrite problem $P_{\gamma, \beta}\left(f, g, z_{0}, w_{0}\right)$ as the following abstract Cauchy problem in $X$ :

$$
\left\{\begin{array}{l}
\left.V^{\prime}(t)+\mathcal{B}^{\gamma, \beta}(V(t)) \ni(f, g), \quad t \in\right] 0, T[ \\
V(0)=\left(z_{0}, w_{0}\right)
\end{array}\right.
$$

A direct consequence of Theorems 3.2 and 3.3 is the following result. 
COROllaRY 3.4 The operator $\mathcal{B}^{\gamma, \beta}$ is $T$-accretive in $X$ and, assuming $\operatorname{Dom}(\gamma)=\mathbb{R}$, and $\operatorname{Dom}(\beta)=\mathbb{R}$ or a smooth, it satisfies the following range condition:

$$
\left\{(\phi, \psi) \in V^{1, p}(\Omega) \times V^{1, p}(\partial \Omega): \int_{\Omega} \phi+\int_{\partial \Omega} \psi \in \mathcal{R}_{\gamma, \beta}\right\} \subset \operatorname{Ran}\left(I+\mathcal{B}^{\gamma, \beta}\right) .
$$

Moreover, we can characterize $\overline{D\left(\mathcal{B}^{\gamma, \beta}\right)} L^{1(\Omega) \times L^{1}(\partial \Omega)}$ as follows.

Theorem 3.5 Under the hypothesis $\operatorname{Dom}(\gamma)=\mathbb{R}$, and $\operatorname{Dom}(\beta)=\mathbb{R}$ or a smooth, we have

$$
{\overline{D\left(\mathcal{B}^{\gamma, \beta}\right)}}^{L^{1}(\Omega) \times L^{1}(\partial \Omega)}=\left\{(z, w) \in L^{1}(\Omega) \times L^{1}(\partial \Omega): \gamma_{-} \leqslant z \leqslant \gamma_{+}, \beta_{-} \leqslant w \leqslant \beta_{+}\right\} .
$$

The proof of this theorem is quite technical and given in the Appendix.

The above results allow us to prove the following theorem concerning mild solutions.

THEOREM 3.6 Let $T>0$. Under the hypothesis $\operatorname{Dom}(\gamma)=\mathbb{R}$, and $\operatorname{Dom}(\beta)=\mathbb{R}$ or a smooth, for any $z_{0} \in L^{1}(\Omega), w_{0} \in L^{1}(\partial \Omega)$ and any $f \in L^{1}\left(0, T ; L^{1}(\Omega)\right), g \in L^{1}\left(0, T ; L^{1}(\partial \Omega)\right)$ satisfying (4) and (6), there exists a unique mild solution of (10).

Proof. For $n \in \mathbb{N}$, let $\epsilon=T / n$, and consider a subdivision $t_{0}=0<t_{1}<\cdots<t_{n-1}<T=t_{n}$ with $t_{i}-t_{i-1}=\epsilon, f_{1}^{\epsilon}, \ldots, f_{n}^{\epsilon} \in L^{p^{\prime}}(\Omega), g_{1}^{\epsilon}, \ldots, g_{n}^{\epsilon} \in L^{p^{\prime}}(\partial \Omega), w_{0}^{\epsilon} \in L^{p^{\prime}}(\Omega), z_{0}^{\epsilon} \in L^{p^{\prime}}(\partial \Omega)$ with

$$
\sum_{i=1}^{n} \int_{t_{i-1}}^{t_{i}}\left(\left\|f(t)-f_{i}^{\epsilon}\right\|_{L^{1}(\Omega)}+\left\|g(t)-g_{i}^{\epsilon}\right\|_{L^{1}(\partial \Omega)}\right) \mathrm{d} t \leqslant \epsilon
$$

and

If we set

$$
\left\|z_{0}^{\epsilon}-z_{0}\right\|_{L^{1}(\Omega)}+\left\|w_{0}^{\epsilon}-w_{0}\right\|_{L^{1}(\partial \Omega)} \leqslant \epsilon .
$$

$$
\left.\left.f_{\epsilon}(t)=f_{i}^{\epsilon}, \quad g_{\epsilon}(t)=g_{i}^{\epsilon} \quad \text { for } t \in\right] t_{i-1}, t_{i}\right], i=1, \ldots, n,
$$

it follows that

$$
\int_{0}^{T}\left(\left\|f(t)-f_{\epsilon}(t)\right\|_{L^{1}(\Omega)}+\left\|g(t)-g_{\epsilon}(t)\right\|_{L^{1}(\partial \Omega)}\right) \mathrm{d} t \leqslant \epsilon .
$$

By Theorem 3.3 applied recursively, for $n$ large enough, there exists a weak solution $\left[u_{i}^{\epsilon}, z_{i}^{\epsilon}, w_{i}^{\epsilon}\right]$ of

$$
\left\{\begin{array}{l}
\gamma\left(u_{i}^{\epsilon}\right)-\epsilon \operatorname{div} \mathbf{a}\left(x, D u_{i}^{\epsilon}\right) \ni \epsilon f_{i}^{\epsilon}+z_{i-1}^{\epsilon}, \\
\epsilon \mathbf{a}\left(x, D u_{i}^{\epsilon}\right) \cdot \eta+\beta\left(u_{i}^{\epsilon}\right) \ni \epsilon g_{i}^{\epsilon}+w_{i-1}^{\epsilon},
\end{array}\right.
$$

for $i=1, \ldots, n$. That is, there exists a unique solution $\left(z_{i}^{\epsilon}, w_{i}^{\epsilon}\right) \in X$ of the time discretized scheme associated with 10$]$,

$$
\left(z_{i}^{\epsilon}, w_{i}^{\epsilon}\right)+\epsilon \mathcal{B}^{\gamma, \beta}\left(z_{i}^{\epsilon}, w_{i}^{\epsilon}\right) \ni \epsilon\left(f_{i}^{\epsilon}, g_{i}^{\epsilon}\right)+\left(z_{i-1}^{\epsilon}, w_{i-1}^{\epsilon}\right) \quad \text { for } i=1, \ldots, n .
$$

Observe that to apply Theorem 3.3 we need to know that 8 holds in every step. Indeed, for the first step this is straightforward. For the other steps, we use the following argument. For each $i=$ $1, \ldots, n$, we have $\left[u_{i}^{\epsilon}, z_{i}^{\epsilon}, w_{i}^{\epsilon}\right] \in W^{1, p}(\Omega) \times V^{1, p}(\Omega) \times V^{1, p}(\partial \Omega)$ and

$$
\int_{\Omega} \mathbf{a}\left(x, D u_{i}^{\epsilon}\right) \cdot D v+\int_{\Omega} \frac{z_{i}^{\epsilon}-z_{i-1}^{\epsilon}}{\epsilon} v+\int_{\partial \Omega} \frac{w_{i}^{\epsilon}-w_{i-1}^{\epsilon}}{\epsilon} v=\int_{\Omega} f_{i}^{\epsilon} v+\int_{\partial \Omega} g_{i}^{\epsilon} v
$$


for all $v \in W^{1, p}(\Omega)$. Therefore, taking $v=1$ in 14 , we have

$$
\int_{\Omega} z_{i}^{\epsilon}+\int_{\partial \Omega} w_{i}^{\epsilon}=\epsilon\left(\int_{\Omega} f_{i}^{\epsilon}+\int_{\partial \Omega} g_{i}^{\epsilon}\right)+\int_{\Omega} z_{i-1}^{\epsilon}+\int_{\partial \Omega} w_{i-1}^{\epsilon}
$$

It follows that

$$
\int_{\Omega} z_{i}^{\epsilon}+\int_{\partial \Omega} w_{i}^{\epsilon}=\epsilon \sum_{j=1}^{i}\left(\int_{\Omega} f_{j}^{\epsilon}+\int_{\partial \Omega} g_{j}^{\epsilon}\right)+\int_{\Omega} z_{0}^{\epsilon}+\int_{\partial \Omega} w_{0}^{\epsilon},
$$

and if we take $n$ large enough, condition $(8)$ is always satisfied.

Therefore, if we define $V_{\epsilon}(t)=\left(z_{\epsilon}(t), w_{\epsilon}(t)\right)$ by

$$
\left\{\begin{array}{l}
z_{\epsilon}(0)=z_{0}^{\epsilon}, \quad w_{\epsilon}(0)=w_{0}^{\epsilon}, \\
\left.\left.z_{\epsilon}(t)=z_{i}^{\epsilon}, \quad w_{\epsilon}(t)=w_{i}^{\epsilon} \quad \text { for } t \in\right] t_{i-1}, t_{i}\right], i=1, \ldots, n,
\end{array}\right.
$$

it is an $\epsilon$-approximate solution of problem $(10)$.

Now by nonlinear semigroup theory (see [10], [14], [25]), on account of Corollary 3.4 and Theorem 3.5, problem (10) has a unique mild solution $V(t)=(z(t), w(t)) \in C([0, T] ; X), z(t)=$ $L^{1}(\Omega)-\lim _{\epsilon \rightarrow 0} z_{\epsilon}(t)$ and $w(t)=L^{1}(\partial \Omega)-\lim _{\epsilon \rightarrow 0} w_{\epsilon}(t)$ uniformly for $t \in[0, T]$.

In principle, it is not clear how these mild solutions have to be interpreted for problem $P_{\gamma, \beta}\left(f, g, z_{0}, w_{0}\right)$. We will see that under the hypothesis of Theorem 2.5 they are weak solutions of $P_{\gamma, \beta}\left(f, g, z_{0}, w_{0}\right)$, which proves the existence part of that theorem.

\section{Existence of weak solutions}

As said in the previous section, the existence part of Theorem 2.5 is shown by proving that the mild solution of problem (10) is a weak solution of problem $P_{\gamma, \beta}\left(f, g, z_{0}, w_{0}\right)$ whenever the assumptions of Theorem 2.5 are fulfilled. Before giving the proof we need to prove some technical lemmas.

\subsection{Preparatory lemmas}

We shall use the following integration by parts lemma in the proof of the existence part and in the proof of the contraction principle. We denote by $(\cdot, \cdot)$ the pairing between $\left(W^{1, p}(\Omega)\right)^{\prime}$ and $W^{1, p}(\Omega)$.

Lemma 4.1 Let $\left.(z, w) \in C\left([0, T] ; L^{1}(\Omega)\right) \times L^{1}(\partial \Omega)\right)$ and $F \in L^{p^{\prime}}\left(0, T ;\left(W^{1, p}(\Omega)\right)^{\prime}\right)$ be such that

$$
\int_{0}^{T} \int_{\Omega} z(t) \psi_{t}+\int_{0}^{T} \int_{\partial \Omega} w(t) \psi_{t}=\int_{0}^{T}(F(t), \psi(t)) \mathrm{d} t
$$

for any $\psi \in W^{1,1}\left(0, T ; W^{1,1}(\Omega) \cap L^{\infty}(\Omega)\right) \cap L^{p}\left(0, T ; W^{1, p}(\Omega)\right)$ with $\psi(0)=\psi(T)=0$. Then

$$
\begin{array}{r}
\int_{0}^{T} \int_{\Omega}\left(\int_{0}^{z(t)} H\left(\cdot,\left(\gamma^{-1}\right)^{0}(s)\right) \mathrm{d} s\right) \psi_{t}+\int_{0}^{T} \int_{\partial \Omega}\left(\int_{0}^{w(t)} H\left(\cdot,\left(\beta^{-1}\right)^{0}(s)\right) \mathrm{d} s\right) \psi_{t} \\
=\int_{0}^{T}(F(t), H(\cdot, u(t)) \psi(t)) \mathrm{d} t,
\end{array}
$$


for any $u \in L^{p}\left(0, T ; W^{1, p}(\Omega)\right)$ with $z \in \gamma(u)$ a.e. in $Q_{T}, w \in \beta(u)$ a.e. in $S_{T}$, for any $\psi \in \mathcal{D}(] 0, T\left[\times \mathbb{R}^{N}\right)$, and for any Carathéodory function $H: \Omega \times \mathbb{R} \rightarrow \mathbb{R}$ such that $H(x, r)$ is nondecreasing in $r, H(\cdot, u) \in L^{p}\left(0, T ; W^{1, p}(\Omega)\right), \int_{0}^{z} H\left(x,\left(\gamma^{-1}\right)^{0}(s)\right) \mathrm{d} s \in L^{1}\left(Q_{T}\right)$ and $\int_{0}^{w} H\left(x,\left(\beta^{-1}\right)^{0}(s)\right) \mathrm{d} s \in L^{1}\left(S_{T}\right)$.

Proof. The proof is similar to the one given in [23] for Dirichlet boundary conditions. We give it here for the sake of completeness.

Let $\psi \in \mathcal{D}(] 0, T\left[\times \mathbb{R}^{N}\right), \psi \geqslant 0$, and for $H_{\tau}=T_{1 / \tau} H, \tau>0$, let

$$
\eta_{\tau}(t)=\frac{1}{\tau} \int_{t}^{t+\tau} H_{\tau}(\cdot, u(s)) \psi(s) \mathrm{d} s .
$$

Then $\eta_{\tau}$ can be used as a test function in (17) and therefore

$$
\begin{aligned}
\int_{0}^{T}\left(F(t), \eta_{\tau}(t)\right) \mathrm{d} t= & \int_{0}^{T} \int_{\Omega} z(t)\left(\eta_{\tau}\right)_{t}+\int_{0}^{T} \int_{\partial \Omega} w(t)\left(\eta_{\tau}\right)_{t} \\
= & \int_{0}^{T} \int_{\Omega} z(t) \frac{H_{\tau}(\cdot, u(t+\tau)) \psi(t+\tau)-H_{\tau}(\cdot, u(t)) \psi(t)}{\tau} \\
& +\int_{0}^{T} \int_{\partial \Omega} w(t) \frac{H_{\tau}(\cdot, u(t+\tau)) \psi(t+\tau)-H_{\tau}(\cdot, u(t)) \psi(t)}{\tau} \\
= & \int_{0}^{T} \int_{\Omega} \frac{z(t-\tau)-z(t)}{\tau} H_{\tau}(\cdot, u(t)) \psi(t) \\
& +\int_{0}^{T} \int_{\partial \Omega} \frac{w(t-\tau)-w(t)}{\tau} H_{\tau}(\cdot, u(t)) \psi(t) .
\end{aligned}
$$

Now, since

$$
\begin{aligned}
& H_{\tau}\left(\cdot, \gamma^{-1}(r)\right) \subset \partial\left(\int_{0}^{r} H_{\tau}\left(\cdot,\left(\gamma^{-1}\right)^{0}(s)\right) \mathrm{d} s\right), \\
& H_{\tau}\left(\cdot, \beta^{-1}(r)\right) \subset \partial\left(\int_{0}^{r} H_{\tau}\left(\cdot,\left(\beta^{-1}\right)^{0}(s)\right) \mathrm{d} s\right), \\
& H_{\tau}(\cdot, u(t)) \in H_{\tau}\left(\cdot, \gamma^{-1}(z(t))\right) \quad \text { a.e. in } \Omega, \\
& H_{\tau}(\cdot, u(t)) \in H_{\tau}\left(\cdot, \beta^{-1}(w(t))\right) \quad \text { a.e. on } \partial \Omega,
\end{aligned}
$$

we have

$$
\begin{array}{cc}
(z(t-\tau)-z(t)) H_{\tau}(\cdot, u(t)) \leqslant \int_{z(t)}^{z(t-\tau)} H_{\tau}\left(\cdot,\left(\gamma^{-1}\right)^{0}(s)\right) \mathrm{d} s & \text { a.e. in } \Omega, \\
(w(t-\tau)-w(t)) H_{\tau}(\cdot, u(t)) \leqslant \int_{w(t)}^{w(t-\tau)} H_{\tau}\left(\cdot,\left(\beta^{-1}\right)^{0}(s)\right) \mathrm{d} s & \text { a.e. on } \partial \Omega .
\end{array}
$$

Therefore

$$
\begin{aligned}
\int_{0}^{T}\left(F(t), \eta_{\tau}(t)\right) \mathrm{d} t \leqslant & \int_{0}^{T} \int_{\Omega} \frac{1}{\tau} \int_{z(t)}^{z(t-\tau)} H_{\tau}\left(\cdot,\left(\gamma^{-1}\right)^{0}(s)\right) \mathrm{d} s \psi(t) \\
& +\int_{0}^{T} \int_{\partial \Omega} \frac{1}{\tau} \int_{w(t)}^{w(t-\tau)} H_{\tau}\left(\cdot,\left(\beta^{-1}\right)^{0}(s)\right) \mathrm{d} s \psi(t)
\end{aligned}
$$




$$
\begin{aligned}
= & \int_{0}^{T} \int_{\Omega} \int_{0}^{z(t)} H_{\tau}\left(\cdot,\left(\gamma^{-1}\right)^{0}(s)\right) \mathrm{d} s \frac{\psi(t+\tau)-\psi(t)}{\tau} \\
& +\int_{0}^{T} \int_{\partial \Omega} \int_{0}^{w(t)} H_{\tau}\left(\cdot,\left(\beta^{-1}\right)^{0}(s)\right) \mathrm{d} s \frac{\psi(t+\tau)-\psi(t)}{\tau} .
\end{aligned}
$$

Letting $\tau \rightarrow 0^{+}$we get

$$
\begin{aligned}
& \int_{0}^{T}(F(t), H(\cdot, u(t)) \psi(t)) \mathrm{d} t \\
& \quad \leqslant \int_{0}^{T} \int_{\Omega}\left(\int_{0}^{z(t)} H\left(x,\left(\gamma^{-1}\right)^{0}(s)\right) \mathrm{d} s\right) \psi_{t}+\int_{0}^{T} \int_{\partial \Omega}\left(\int_{0}^{w(t)} H\left(x,\left(\beta^{-1}\right)^{0}(s)\right) \mathrm{d} s\right) \psi_{t} .
\end{aligned}
$$

Taking now $\tilde{\eta}_{\tau}(t)=\frac{1}{\tau} \int_{t}^{t+\tau} H_{\tau}(\cdot, u(s-\tau)) \psi(s) \mathrm{d} s$, and arguing as above we get the other inequality.

To prove the existence of weak solutions we shall also use the following result.

LEMMA 4.2 Let $\left\{u_{n}\right\}_{n \in \mathbb{N}} \subset W^{1, p}(\Omega),\left\{z_{n}\right\}_{n \in \mathbb{N}} \subset L^{1}(\Omega),\left\{w_{n}\right\}_{n \in \mathbb{N}} \subset L^{1}(\partial \Omega)$ be such that, for every $n \in \mathbb{N}, z_{n} \in \gamma\left(u_{n}\right)$ a.e. in $\Omega$ and $w_{n} \in \beta\left(u_{n}\right)$ a.e. in $\partial \Omega$. Suppose that

(i) if $\mathcal{R}_{\gamma, \beta}^{+}=+\infty$, there exists $M>0$ such that

$$
\int_{\Omega} z_{n}^{+}+\int_{\partial \Omega} w_{n}^{+}<M \quad \forall n \in \mathbb{N}
$$

(ii) if $\mathcal{R}_{\gamma, \beta}^{+}<+\infty$, there exist $M \in \mathbb{R}$ and $h>0$ such that

$$
\int_{\Omega} z_{n}+\int_{\partial \Omega} w_{n}<M<\mathcal{R}_{\gamma, \beta}^{+} \quad \forall n \in \mathbb{N}
$$

and

$$
\max \left\{\int_{\left\{x \in \Omega: z_{n}(x)<-h\right\}}\left|z_{n}\right|, \int_{\left\{x \in \partial \Omega: w_{n}(x)<-h\right\}}\left|w_{n}\right|\right\}<\frac{\mathcal{R}_{\gamma, \beta}^{+}-M}{8} \quad \forall n \in \mathbb{N} .
$$

Then there exists a constant $C=C(M)$ in case (i), and $C=C(M, h)$ in case (ii), such that

$$
\left\|u_{n}^{+}\right\|_{L^{p}(\Omega)} \leqslant C\left(\left\|D u_{n}^{+}\right\|_{L^{p}(\Omega)}+1\right) \quad \forall n \in \mathbb{N} .
$$

In order to prove Lemma 4.2, we use the following well known result (see [53]).

LEMmA 4.3 (i) There exists a constant $C(\Omega, N, p)$ such that, for any $K \subset \Omega$ with $|K|>0$,

$$
\|u\|_{L^{p}(\Omega)} \leqslant \frac{C(\Omega, N, p)}{|K|^{1 / p}}\left(\|D u\|_{L^{p}(\Omega)}+\|u\|_{L^{p}(K)}\right) \quad \forall u \in W^{1, p}(\Omega) .
$$

(ii) There exists a constant $\hat{C}(\Omega, N, p)$ such that, for any $\Gamma \subset \partial \Omega$ with $|\Gamma|>0$,

$$
\|u\|_{L^{p}(\Omega)} \leqslant \frac{\hat{C}(\Omega, N, p)}{|\Gamma|^{1 / p}}\left(\|D u\|_{L^{p}(\Omega)}+\|u\|_{L^{p}(\Gamma)}\right) \quad \forall u \in W^{1, p}(\Omega) .
$$


Proof of Lemma 4.2 Assume first that $\mathcal{R}_{\gamma, \beta}^{+}=+\infty$. Then $\gamma^{+}=+\infty$ or $\beta^{+}=+\infty$. Suppose first that $\gamma^{+}=+\infty$. Then, by assumption, there exists $M>0$ such that

$$
\int_{\Omega} z_{n}^{+}<M \quad \forall n \in \mathbb{N}
$$

Let $K_{n}=\left\{x \in \Omega: z_{n}^{+}(x)<2 M /|\Omega|\right\}$ for every $n \in \mathbb{N}$. Then

$$
0 \leqslant \int_{K_{n}} z_{n}^{+}=\int_{\Omega} z_{n}^{+}-\int_{\Omega \backslash K_{n}} z_{n}^{+} \leqslant M-\left(|\Omega|-\left|K_{n}\right|\right) \frac{2 M}{|\Omega|}=\left|K_{n}\right| \frac{2 M}{|\Omega|}-M .
$$

Therefore, $\left|K_{n}\right| \geqslant|\Omega| / 2$, and

$$
\left\|u_{n}^{+}\right\|_{L^{p}\left(K_{n}\right)} \leqslant\left|K_{n}\right|^{1 / p} \sup \gamma^{-1}\left(\frac{2 M}{|\Omega|}\right) .
$$

Then, by Lemma 4.3 , for all $n \in \mathbb{N}$,

$$
\left\|u_{n}^{+}\right\|_{L^{p}(\Omega)} \leqslant C(\Omega, N, p)\left(\left(\frac{2}{|\Omega|}\right)^{1 / p}\left\|D u_{n}^{+}\right\|_{L^{p}(\Omega)}+\sup \gamma^{-1}\left(\frac{2 M}{|\Omega|}\right)\right) .
$$

If $\beta^{+}=+\infty$, we similarly get, for all $n \in \mathbb{N}$,

$$
\left\|u_{n}^{+}\right\|_{L^{p}(\Omega)} \leqslant \hat{C}(\Omega, N, p)\left(\left(\frac{2}{|\partial \Omega|}\right)^{1 / p}\left\|D u_{n}^{+}\right\|_{L^{p}(\Omega)}+\sup \beta^{-1}\left(\frac{2 M}{|\partial \Omega|}\right)\right),
$$

where $\hat{C}(\Omega, N, p)$ is given in Lemma 4.3

Now assume that $\mathcal{R}_{\gamma, \beta}^{+}<+\infty$, and let $\delta=\mathcal{R}_{\gamma, \beta}^{+}-M$. Then, by assumption,

$$
\int_{\Omega} z_{n}+\int_{\partial \Omega} w_{n}<\mathcal{R}_{\gamma, \beta}^{+}-\delta
$$

Consequently, for every $n \in \mathbb{N}$,

$$
\int_{\Omega} z_{n}<\gamma^{+}|\Omega|-\frac{\delta}{2}
$$

or

$$
\int_{\partial \Omega} w_{n}<\beta^{+}|\partial \Omega|-\frac{\delta}{2} \text {. }
$$

For $n \in \mathbb{N}$ such that 20 holds, let $K_{n}=\left\{x \in \Omega: z_{n}(x)<\gamma^{+}-\delta /(4|\Omega|)\right\}$. Then, on the one hand,

$$
\int_{K_{n}} z_{n}=\int_{\Omega} z_{n}-\int_{\Omega \backslash K_{n}} z_{n}<-\frac{\delta}{4}+\left|K_{n}\right|\left(\gamma^{+}-\frac{\delta}{4|\Omega|}\right),
$$

and, on the other hand,

$$
\int_{K_{n}} z_{n}=-\int_{K_{n} \cap\left\{x \in \Omega: z_{n}<-h\right\}}\left|z_{n}\right|+\int_{K_{n} \cap\left\{x \in \Omega: z_{n} \geqslant-h\right\}} z_{n} \geqslant-\frac{\delta}{8}-h\left|K_{n}\right| .
$$


Therefore,

$$
\left|K_{n}\right|\left(h-\frac{\delta}{4|\Omega|}+\gamma^{+}\right) \geqslant \frac{\delta}{8} .
$$

Hence $\left|K_{n}\right|>0, h-\delta /(4|\Omega|)+\gamma^{+}>0$ and

$$
\left|K_{n}\right| \geqslant \frac{\delta / 8}{h-\delta /(4|\Omega|)+\gamma^{+}} .
$$

Consequently,

$$
\left\|u_{n}^{+}\right\|_{L^{p}\left(K_{n}\right)} \leqslant\left|K_{n}\right|^{1 / p} \sup \gamma^{-1}\left(\gamma^{+}-\frac{\delta}{4|\Omega|}\right) .
$$

Then, by Lemma 4.3 .

$$
\left\|u_{n}^{+}\right\|_{L^{p}(\Omega)} \leqslant C(\Omega, N, p)\left(\left(\frac{h-\delta /(4|\Omega|)+\gamma^{+}}{\delta / 8}\right)^{1 / p}\left\|D u_{n}^{+}\right\|_{L^{p}(\Omega)}+\sup \gamma^{-1}\left(\gamma^{+}-\frac{\delta}{4|\Omega|}\right)\right) .
$$

Similarly, for $n \in \mathbb{N}$ such that 21 holds, we get $\left|\left\{x \in \partial \Omega: w_{n}(x)<\beta^{+}-\delta /(4|\partial \Omega|)\right\}\right|>0$, $h-\delta /(4|\partial \Omega|)+\beta^{+}>0$ and

$$
\left\|u_{n}^{+}\right\|_{L^{p}(\Omega)} \leqslant \hat{C}(\Omega, N, p)\left(\left(\frac{h-\delta /(4|\partial \Omega|)+\beta^{+}}{\delta / 8}\right)^{1 / p}\left\|D u_{n}^{+}\right\|_{L^{p}(\Omega)}+\sup \beta^{-1}\left(\beta^{+}-\frac{\delta}{4|\partial \Omega|}\right)\right)
$$

where $\hat{C}(\Omega, N, p)$ is given in Lemma 4.3

\subsection{Proof of the existence part of Theorem 2.5}

Let $T>0$. Let $f \in L^{p^{\prime}}\left(0, T ; L^{p^{\prime}}(\Omega)\right), g \in L^{p^{\prime}}\left(0, T ; L^{p^{\prime}}(\partial \Omega)\right), z_{0} \in L^{p^{\prime}}(\Omega)$ and $w_{0} \in L^{p^{\prime}}(\partial \Omega)$ satisfy (4)-6). Let $V(t)=(z(t), w(t))$ be the mild solution of problem (10) given by Theorem 3.6 . Our aim is to prove that $(z, w)$ is a weak solution of problem $P_{\gamma, \beta}\left(f, g, z_{0}, w_{0}\right)$.

Following the proof of the existence of the mild solution (Theorem 3.6), for $n \in \mathbb{N}$, let $\epsilon=T / n$, and consider a subdivision $t_{0}=0<t_{1}<\cdots<t_{n-1}<T=t_{n}$ with $t_{i}-t_{i-1}=\epsilon$ and $f_{1}, \ldots, f_{n} \in$ $L^{p^{\prime}}(\Omega), g_{1}, \ldots, g_{n} \in L^{p^{\prime}}(\partial \Omega)$ with

$$
\sum_{i=1}^{n} \int_{t_{i-1}}^{t_{i}}\left(\left\|f(t)-f_{i}\right\|_{L^{p^{\prime}(\Omega)}}^{p^{\prime}}+\left\|g(t)-g_{i}\right\|_{L^{p^{\prime}(\partial \Omega)}}^{p^{\prime}}\right) \mathrm{d} t \leqslant \epsilon .
$$

Then

$$
\begin{array}{ll}
z(t)=L^{1}(\Omega)-\lim _{\epsilon \rightarrow 0} z_{\epsilon}(t) & \text { uniformly for } t \in[0, T], \\
w(t)=L^{1}(\partial \Omega)-\lim _{\epsilon \rightarrow 0} w_{\epsilon}(t) & \text { uniformly for } t \in[0, T],
\end{array}
$$

where $z_{\epsilon}(t)$ and $w_{\epsilon}(t)$ are given, for $\epsilon$ small enough, by

$$
\left\{\begin{array}{lll}
z_{\epsilon}(t)=z_{0}, & w_{\epsilon}(t)=w_{0} & \text { for } t \in]-\infty, 0] \\
z_{\epsilon}(t)=z_{i}, & w_{\epsilon}(t)=w_{i} & \text { for } \left.t \in] t_{i-1}, t_{i}\right], i=1, \ldots, n
\end{array}\right.
$$


$\left[u_{i}, z_{i}, w_{i}\right] \in W^{1, p}(\Omega) \times V^{1, p}(\Omega) \times V^{1, p}(\partial \Omega)$ being such that $z_{i}(x) \in \gamma\left(u_{i}(x)\right)$ a.e. in $\Omega$, $w_{i}(x) \in \beta\left(u_{i}(x)\right)$ a.e. in $\partial \Omega$ and

$$
\int_{\Omega} \mathbf{a}\left(x, D u_{i}\right) \cdot D v+\int_{\Omega} \frac{z_{i}-z_{i-1}}{\epsilon} v+\int_{\partial \Omega} \frac{w_{i}-w_{i-1}}{\epsilon} v=\int_{\Omega} f_{i} v+\int_{\partial \Omega} g_{i} v
$$

for all $v \in W^{1, p}(\Omega)$.

Taking $v=u_{i}$ as a test function in 24, we get

$$
\int_{\Omega} \mathbf{a}\left(x, D u_{i}\right) \cdot D u_{i}+\int_{\Omega} \frac{z_{i}-z_{i-1}}{\epsilon} u_{i}+\int_{\partial \Omega} \frac{w_{i}-w_{i-1}}{\epsilon} u_{i}=\int_{\Omega} f_{i} u_{i}+\int_{\partial \Omega} g_{i} u_{i} .
$$

Since $z_{i}(x) \in \gamma\left(u_{i}(x)\right)$ a.e. in $\Omega$ and $w_{i}(x) \in \beta\left(u_{i}(x)\right)$ a.e. in $\partial \Omega$, we have

$$
\begin{array}{ll}
u_{i}(x) \in \gamma^{-1}\left(z_{i}(x)\right)=\partial j_{\gamma}^{*}\left(z_{i}(x)\right) & \text { a.e. in } \Omega, \\
u_{i}(x) \in \beta^{-1}\left(w_{i}(x)\right)=\partial j_{\beta}^{*}\left(w_{i}(x)\right) & \text { a.e. in } \partial \Omega
\end{array}
$$

Hence,

$$
\begin{array}{cc}
j_{\gamma}^{*}\left(z_{i-1}(x)\right)-j_{\gamma}^{*}\left(z_{i}(x)\right) \geqslant\left(z_{i-1}(x)-z_{i}(x)\right) u_{i}(x) & \text { a.e. in } \Omega, \\
j_{\beta}^{*}\left(w_{i-1}(x)\right)-j_{\beta}^{*}\left(w_{i}(x)\right) \geqslant\left(w_{i-1}(x)-w_{i}(x)\right) u_{i}(x) & \text { a.e. in } \partial \Omega
\end{array}
$$

Therefore,

$$
\frac{1}{\epsilon} \int_{\Omega}\left(j_{\gamma}^{*}\left(z_{i}\right)-j_{\gamma}^{*}\left(z_{i-1}\right)\right)+\frac{1}{\epsilon} \int_{\partial \Omega}\left(j_{\beta}^{*}\left(w_{i}\right)-j_{\beta}^{*}\left(w_{i-1}\right)\right) \leqslant \int_{\Omega} \frac{z_{i}-z_{i-1}}{\epsilon} u_{i}+\int_{\partial \Omega} \frac{w_{i}-w_{i-1}}{\epsilon} u_{i},
$$

and by $(25)$, we get

$\int_{\Omega} \mathbf{a}\left(x, D u_{i}\right) \cdot D u_{i}+\frac{1}{\epsilon} \int_{\Omega}\left(j_{\gamma}^{*}\left(z_{i}\right)-j_{\gamma}^{*}\left(z_{i-1}\right)\right)+\frac{1}{\epsilon} \int_{\partial \Omega}\left(j_{\beta}^{*}\left(w_{i}\right)-j_{\beta}^{*}\left(w_{i-1}\right)\right) \leqslant \int_{\Omega} f_{i} u_{i}+\int_{\partial \Omega} g_{i} u_{i}$.

Then, integrating in time and adding in the last inequality, we obtain that

$$
\begin{aligned}
\sum_{i=1}^{n} \int_{t_{i-1}}^{t_{i}} \int_{\Omega} \mathbf{a}\left(x, D u_{i}\right) \cdot D u_{i}+\int_{\Omega}\left(j_{\gamma}^{*}\left(z_{n}\right)-j_{\gamma}^{*}\left(z_{0}\right)\right) & +\int_{\partial \Omega}\left(j_{\beta}^{*}\left(w_{n}\right)-j_{\beta}^{*}\left(w_{0}\right)\right) \\
& \leqslant \sum_{i=1}^{n} \int_{t_{i-1}}^{t_{i}}\left(\int_{\Omega} f_{i} u_{i}+\int_{\partial \Omega} g_{i} u_{i}\right) .
\end{aligned}
$$

Consequently, if we set $f_{\epsilon}(t)=f_{i}, g_{\epsilon}(t)=g_{i}$ and $u_{\epsilon}(t)=u_{i}$ for $\left.\left.t \in\right] t_{i-1}, t_{i}\right], i=1, \ldots, n$, it follows that

$$
\begin{array}{r}
\int_{0}^{T} \int_{\Omega} \mathbf{a}\left(x, D u_{\epsilon}(t)\right) \cdot D u_{\epsilon}(t) \mathrm{d} t+\int_{\Omega}\left(j_{\gamma}^{*}\left(z_{n}\right)-j_{\gamma}^{*}\left(z_{0}\right)\right)+\int_{\partial \Omega}\left(j_{\beta}^{*}\left(w_{n}\right)-j_{\beta}^{*}\left(w_{0}\right)\right) \\
\leqslant \int_{0}^{T} \int_{\Omega} f_{\epsilon}(t) u_{\epsilon}(t)+\int_{0}^{T} \int_{\partial \Omega} g_{\epsilon}(t) u_{\epsilon}(t) .
\end{array}
$$


Then, having in mind $\left(\mathrm{H}_{1}\right)$ and $(5)$, we see that there exists a positive constant $C_{1}$ such that

$$
\begin{aligned}
\lambda \int_{0}^{T} \int_{\Omega}\left|D u_{\epsilon}(t)\right|^{p} \mathrm{~d} t \leqslant \int_{0}^{T} \int_{\Omega} \mathbf{a}\left(x, D u_{\epsilon}(t)\right) \cdot D u_{\epsilon}(t) \mathrm{d} t \\
\quad \leqslant \int_{\Omega} j_{\gamma}^{*}\left(z_{0}\right)+\int_{\partial \Omega} j_{\beta}^{*}\left(w_{0}\right)+\int_{0}^{T} \int_{\Omega} f_{\epsilon}(t) u_{\epsilon}(t)+\int_{0}^{T} \int_{\partial \Omega} g_{\epsilon}(t) u_{\epsilon}(t) \\
\leqslant C_{1}+\int_{0}^{T}\left\|f_{\epsilon}(t)\right\|_{L^{p^{\prime}}(\Omega)}\left\|u_{\epsilon}(t)\right\|_{L^{p}(\Omega)} \mathrm{d} t+\int_{0}^{T}\|g\|_{L^{p^{\prime}(\partial \Omega)}}\left\|u_{\epsilon}(t)\right\|_{L^{p}(\partial \Omega)} \mathrm{d} t .
\end{aligned}
$$

Therefore, using Young's inequality, for any $\mu>0$ there exists $C_{2}(\mu)>0$ such that

$$
\int_{0}^{T} \int_{\Omega}\left|D u_{\epsilon}(t)\right|^{p} \mathrm{~d} t \leqslant C_{2}(\mu)+\mu \int_{0}^{T}\left(\left\|u_{\epsilon}(t)\right\|_{L^{p}(\Omega)}^{p}+\left\|u_{\epsilon}(t)\right\|_{L^{p}(\partial \Omega)}^{p}\right) \mathrm{d} t .
$$

Hence, by the trace theorem, for any $\mu>0$ there exists $C_{3}(\mu)>0$ such that

$$
\int_{0}^{T} \int_{\Omega}\left|D u_{\epsilon}(t)\right|^{p} \mathrm{~d} t \leqslant C_{3}(\mu)+\mu \int_{0}^{T}\left(\left\|u_{\epsilon}(t)\right\|_{L^{p}(\Omega)}^{p}+\left\|D u_{\epsilon}(t)\right\|_{L^{p}(\Omega)}^{p}\right) \mathrm{d} t .
$$

By 22, , if $\mathcal{R}_{\gamma, \beta}^{+}=+\infty$, there exist $M>0$ and $n_{0} \in \mathbb{N}$ such that

$$
\sup _{t \in[0, T]} \int_{\Omega} z_{\epsilon}^{+}(t)+\int_{\partial \Omega} w_{\epsilon}^{+}(t)<M \quad \forall n \geqslant n_{0}
$$

and if $\mathcal{R}_{\gamma, \beta}^{+}<+\infty$, there exist $M \in \mathbb{R}, h>0$ and $n_{0} \in \mathbb{N}$ such that, for all $n \geqslant n_{0}$,

$$
\sup _{t \in[0, T]} \int_{\Omega} z_{\epsilon}(t)+\int_{\partial \Omega} w_{\epsilon}(t)<M<\mathcal{R}_{\gamma, \beta}^{+}
$$

and

$$
\sup _{t \in[0, T]} \max \left\{\int_{\left\{x \in \Omega: z_{\epsilon}(t)(x)<-h\right\}}\left|z_{\epsilon}(t)\right|, \int_{\left\{x \in \partial \Omega: w_{\epsilon}(t)(x)<-h\right\}}\left|w_{\epsilon}(t)\right|\right\}<\frac{\mathcal{R}_{\gamma, \beta}^{+}-M}{8} .
$$

Consequently, from Lemma 4.2 , there exists a constant $C_{4}>0$ such that

$$
\left\|u_{\epsilon}^{+}(t)\right\|_{L^{p}(\Omega)} \leqslant C_{4}\left(\left\|D u_{\epsilon}^{+}(t)\right\|_{L^{p}(\Omega)}+1\right) \quad \text { for all } t \in[0, T]
$$

Similarly, there exists $C_{5}>0$ such that

$$
\left\|u_{\epsilon}^{-}(t)\right\|_{L^{p}(\Omega)} \leqslant C_{5}\left(\left\|D u_{\epsilon}^{-}(t)\right\|_{L^{p}(\Omega)}+1\right) \quad \text { for all } t \in[0, T] .
$$

Consequently, from 27), 28, and 29, choosing $\mu$ small enough, we deduce that there exists $C_{6}>0$ such that

$$
\int_{0}^{T} \int_{\Omega}\left|D u_{\epsilon}(t)\right|^{p} \mathrm{~d} t \leqslant C_{6} .
$$


By 30,28 and $29,\left\{u_{\epsilon}\right\}$ is bounded in $L^{p}\left(0, T ; W^{1, p}(\Omega)\right)$. So, there exists a subsequence, denoted again $\left\{u_{\epsilon}\right\}$, such that

$$
\begin{array}{ll}
u_{\epsilon} \rightarrow u & \text { weakly in } L^{p}\left(0, T ; W^{1, p}(\Omega)\right) \text { as } \epsilon \rightarrow 0^{+}, \\
u_{\epsilon} \rightarrow u & \text { weakly in } L^{p}\left(S_{T}\right) \text { as } \epsilon \rightarrow 0^{+} .
\end{array}
$$

Since $z_{\epsilon} \in \gamma\left(u_{\epsilon}\right)$ a.e. in $Q_{T}, w_{\epsilon} \in \beta\left(u_{\epsilon}\right)$ a.e. on $S_{T}, z_{\epsilon} \rightarrow z$ in $L^{1}\left(Q_{T}\right)$ and $w_{\epsilon} \rightarrow w$ in $L^{1}\left(S_{T}\right)$, having in mind 31 , 32 and using a monotonicity argument we conclude that $z \in \gamma(u)$ a.e. in $Q_{T}$ and $w \in \beta(u)$ a.e. on $S_{T}$.

Since $\left\{D u_{\epsilon}\right\}$ is bounded in $L^{p}\left(Q_{T}\right)$, by $\left(\mathrm{H}_{2}\right)$ the sequence $\left\{\left|\mathbf{a}\left(x, D u_{\epsilon}\right)\right|\right\}$ is bounded in $L^{p^{\prime}}\left(Q_{T}\right)$, so we can assume that

$$
\mathbf{a}\left(x, D u_{\epsilon}\right) \rightarrow \Phi \quad \text { weakly in } L^{p^{\prime}}\left(Q_{T}\right) \text { as } \epsilon \rightarrow 0^{+} .
$$

From (24), we have

$$
\begin{aligned}
\int_{\Omega} \mathbf{a}\left(x, D u_{\epsilon}(t)\right) \cdot D v+\int_{\Omega} \frac{z_{\epsilon}(t)-z_{\epsilon}(t-\epsilon)}{\epsilon} v+\int_{\partial \Omega} \frac{w_{\epsilon}(t)-w_{\epsilon}(t-\epsilon)}{\epsilon} v & =\int_{\Omega} f_{\epsilon}(t) v+\int_{\partial \Omega} g_{\epsilon}(t) v
\end{aligned}
$$

for all $v \in W^{1, p}(\Omega)$. Then, given $\psi \in W^{1,1}\left(0, T ; W^{1,1}(\Omega) \cap L^{\infty}(\Omega)\right) \cap L^{p}\left(0, T ; W^{1, p}(\Omega)\right)$, $\psi(0)=\psi(T)=0$, from (34), we get

$$
\begin{aligned}
\int_{0}^{T} \int_{\Omega} \mathbf{a}(x, D & \left.u_{\epsilon}(t)\right) \cdot D \psi+\int_{\Omega} \int_{0}^{T} \frac{z_{\epsilon}(t)-z_{\epsilon}(t-\epsilon)}{\epsilon} \psi(t) \\
& +\int_{\partial \Omega} \int_{0}^{T} \frac{w_{\epsilon}(t)-w_{\epsilon}(t-\epsilon)}{\epsilon} \psi(t)=\int_{0}^{T} \int_{\Omega} f_{\epsilon}(t) \psi+\int_{0}^{T} \int_{\partial \Omega} g_{\epsilon}(t) \psi
\end{aligned}
$$

Now,

$$
\begin{aligned}
\lim _{\epsilon \rightarrow 0} & \int_{\Omega} \int_{0}^{T} \frac{z_{\epsilon}(t)-z_{\epsilon}(t-\epsilon)}{\epsilon} \psi(t) \\
& =\lim _{\epsilon \rightarrow 0}\left(-\int_{\Omega} \int_{0}^{T-\epsilon} z_{\epsilon}(t) \frac{\psi(t+\epsilon)-\psi(t)}{\epsilon}+\int_{\Omega} \int_{T-\epsilon}^{T} \frac{z_{\epsilon}(t) \psi(t)}{\epsilon}-\int_{\Omega} \int_{0}^{\epsilon} \frac{z_{0} \psi(t)}{\epsilon}\right) \\
& =-\int_{0}^{T} \int_{\Omega} z(t) \psi_{t} .
\end{aligned}
$$

Similarly,

$$
\lim _{\epsilon \rightarrow 0} \int_{\partial \Omega} \int_{0}^{T} \frac{w_{\epsilon}(t)-w_{\epsilon}(t-\epsilon)}{\epsilon} \psi(t)=-\int_{0}^{T} \int_{\partial \Omega} w(t) \psi_{t} .
$$

Therefore, taking the limit in 35 as $\epsilon \rightarrow 0^{+}$, we obtain

$$
\int_{0}^{T} \int_{\Omega} \Phi \cdot D \psi-\int_{0}^{T} \int_{\Omega} z(t) \psi_{t}-\int_{0}^{T} \int_{\partial \Omega} w(t) \psi_{t}=\int_{0}^{T} \int_{\Omega} f(t) \psi+\int_{0}^{T} \int_{\partial \Omega} g(t) \psi
$$


Thus, to finish the proof of the existence, we only need to show that $\Phi=\mathbf{a}(x, D u)$. To do that we prove the following inequality:

$$
\limsup _{\epsilon \rightarrow 0} \int_{Q_{T}} \mathbf{a}\left(x, D u_{\epsilon}\right) \cdot D u_{\epsilon} \leqslant \int_{Q_{T}} \Phi \cdot D u,
$$

and we apply Minty-Browder's method. Indeed, if we assume that 37 holds, then for any $\rho \in$ $L^{p}\left(0, T ; W^{1, p}(\Omega)\right)$,

$$
\int_{Q_{T}} \mathbf{a}(x, D \rho) \cdot D\left(u_{\epsilon}-\rho\right) \leqslant \int_{Q_{T}} \mathbf{a}\left(x, D u_{\epsilon}\right) \cdot D\left(u_{\epsilon}-\rho\right),
$$

so that, passing to the limit and using (37), we get

$$
\int_{Q_{T}} \mathbf{a}(x, D \rho) \cdot D(u-\rho) \leqslant \int_{Q_{T}} \Phi \cdot D(u-\rho) .
$$

Then taking $\rho=u \pm \lambda \xi$ for $\lambda>0$ and $\xi \in L^{p}\left(0, T ; W^{1, p}(\Omega)\right)$, we get

$$
\int_{Q_{T}} \mathbf{a}(x, D(u+\lambda \rho)) \cdot D \xi=\int_{Q_{T}} \Phi \cdot D \xi
$$

and by letting $\lambda \rightarrow 0$, we obtain

$$
\int_{Q_{T}} \mathbf{a}(x, D(u)) \cdot D \xi=\int_{Q_{T}} \Phi \cdot D \xi \quad \text { for any } \xi \in L^{p}\left(0, T ; W^{1, p}(\Omega)\right),
$$

which implies that $\mathbf{a}(x, D(u))=\Phi$ a.e. in $Q$.

Now, let us prove (37). Thanks to 26) and Fatou's lemma, we have

$$
\begin{aligned}
\limsup _{\epsilon \rightarrow 0} \int_{0}^{T} \int_{\Omega} \mathbf{a}\left(x, D u_{\epsilon}(t)\right) & \cdot D u_{\epsilon}(t) \mathrm{d} t \leqslant-\int_{\Omega}\left(j_{\gamma}^{*}(z(T))-j_{\gamma}^{*}\left(z_{0}\right)\right) \\
& -\int_{\partial \Omega}\left(j_{\beta}^{*}(w(T))-j_{\beta}^{*}\left(w_{0}\right)\right)+\int_{0}^{T} \int_{\Omega} f u+\int_{0}^{T} \int_{\partial \Omega} g u .
\end{aligned}
$$

On the other hand, (36) can be rewritten as

$$
\int_{0}^{T} \int_{\Omega} z(t) \psi_{t}+\int_{0}^{T} \int_{\partial \Omega} w(t) \psi_{t}=\int_{0}^{T}(F(t), \psi(t)) \mathrm{d} t
$$

where $F$ is given by

$$
(F(t), \psi(t))=\int_{\Omega} \Phi(t) \cdot D \psi(t)-\int_{\Omega} f(t) \psi(t)-\int_{\partial \Omega} g(t) \psi(t) .
$$

Then, by Lemma 4.1 applied to the above $F, H(x, r)=r$ and $\psi(t, x)=\xi(x) \phi(t), \xi \in \mathcal{D}\left(\mathbb{R}^{N}\right)$, $\xi=1$ in $\Omega, \phi \in \mathcal{D}(] 0, T[$, we get

$$
-\frac{\mathrm{d}}{\mathrm{d} t} \int_{\Omega} j_{\gamma}^{*}(z)-\frac{\mathrm{d}}{\mathrm{d} t} \int_{\partial \Omega} j_{\beta}^{*}(w)=(F, u) \quad \text { in } \mathcal{D}^{\prime}(] 0, T[) .
$$


Therefore,

$$
\int_{\Omega} j_{\gamma}^{*}(z)+\int_{\partial \Omega} j_{\beta}^{*}(w) \in W^{1,1}(] 0, T[) .
$$

So, integrating from 0 to $T$ in 39 , we have

$$
\begin{aligned}
\int_{0}^{T} \int_{\Omega} \Phi \cdot D u= & -\int_{\Omega}\left(j_{\gamma}^{*}(z(T))-j_{\gamma}^{*}\left(z_{0}\right)\right)-\int_{\partial \Omega}\left(j_{\beta}^{*}(w(T))-j_{\beta}^{*}\left(w_{0}\right)\right) \\
& +\int_{0}^{T} \int_{\Omega} f u+\int_{0}^{T} \int_{\partial \Omega} g u .
\end{aligned}
$$

Hence, using 38, we obtain 37.

\section{Contraction principle}

Our main tool to prove the contraction principle is the concept of integral solution due to $\mathrm{Ph}$. Bénilan (see [10], [14]).

DEFINITION 5.1 A function $V=(z, w) \in C([0, T] ; X)$ is an integral solution of $[10]$ in $[0, T]$ if for every $(\hat{f}, \hat{g}) \in \mathcal{B}^{\gamma, \beta}(\hat{z}, \hat{w})$, we have

$$
\begin{aligned}
\frac{\mathrm{d}}{\mathrm{d} t} \int_{\Omega}|z(t)-\hat{z}|+\frac{\mathrm{d}}{\mathrm{d} t} \int_{\partial \Omega} & |w(t)-\hat{w}| \\
\leqslant & \int_{\Omega}(f(t)-\hat{f}) \operatorname{sign}_{0}(z(t)-\hat{z})+\int_{\{x \in \Omega: z(t)=\hat{z}\}}|f(t)-\hat{f}| \\
& +\int_{\partial \Omega}(g(t)-\hat{g}) \operatorname{sign}_{0}(w(t)-\hat{w})+\int_{\{x \in \partial \Omega: w(t)=\hat{w}\}}|g(t)-\hat{g}|
\end{aligned}
$$

in $\mathcal{D}^{\prime}(] 0, T[)$, and $V(0)=\left(z_{0}, w_{0}\right)$.

Since $\mathcal{B}^{\gamma, \beta}$ is accretive in $X$, it is well known (see [10], [14]) that mild solutions and integral solutions of problem (10) coincide and a contraction principle holds. We shall prove in Theorem 5.3 that a weak solution of $P_{\gamma, \beta}\left(f, g, z_{0}, w_{0}\right)$ in $[0, T]$ is an integral solution of (10). Consequently, since, in fact, $\mathcal{B}^{\gamma, \beta}$ is $T$-accretive in $X$, the contraction principle 3 given in Theorem 2.4 follows.

In the proof of Theorem 5.3 the main difficulties are due to the nonlinear and nonhomogeneous boundary conditions and to the jumps of $\gamma$ and $\beta$. In [18], to obtain the $L^{1}$-contraction principle for a similar problem in the case $\beta=\{0\} \times \mathbb{R}$, and for $\gamma$ having a set of jumps without density points, the authors give an improvement of the "hole filling" argument of [22] and use the doubling variable technique. This technique can be adapted to our problem. Now, by nonlinear semigroup theory, we are able to simplify the proof without using the doubling variable technique and without imposing any condition on the jumps of $\gamma$ and $\beta$.

LEMMA 5.2 Let $(z, w)$ be a weak solution of problem $P_{\gamma, \beta}\left(f, g, z_{0}, w_{0}\right)$ in $[0, T]$. Let $u \in$ $L^{p}\left(0, T ; W^{1, p}(\Omega)\right)$ be such that $z \in \gamma(u)$ a.e. in $Q_{T}$ and $w \in \beta(u)$ a.e. on $S_{T}$ as in Definition 2.2. Let $\hat{z}, \hat{f} \in L^{1}(\Omega)$ and $\hat{u} \in W^{1, p}(\Omega)$ with $\hat{z} \in \gamma(\hat{u})$ a.e. in $\Omega$ be such that

$$
\int_{\Omega} \mathbf{a}(x, D \hat{u}) \cdot D \psi=\int_{\Omega} \hat{f} \psi \quad \forall \psi \in W_{0}^{1, p}(\Omega) \cap L^{\infty}(\Omega) .
$$


Then, for any $\psi \in \mathcal{D}(\Omega), \psi \geqslant 0$,

$$
\begin{aligned}
\frac{\mathrm{d}}{\mathrm{d} t} \int_{\Omega}|z(t)-\hat{z}| \psi+\int_{\Omega} \operatorname{sign}_{0}(u(t)-\hat{u})(\mathbf{a}(x, D u(t))-\mathbf{a}(x, D \hat{u})) \cdot D \psi & \\
& \leqslant \int_{\Omega}(f(t)-\hat{f}) \operatorname{sign}_{0}(z(t)-\hat{z}) \psi+\int_{\{x \in \Omega: z(t)=\hat{z}\}}|f(t)-\hat{f}| \psi
\end{aligned}
$$

in $\mathcal{D}^{\prime}(] 0, T[)$.

Proof. In Lemma 4.1, take the function $F$ given by

$$
(F(t), \psi(t))=\int_{\Omega} \mathbf{a}(x, D u(t)) \cdot D \psi(t)-\int_{\Omega} f(t) \psi(t)-\int_{\partial \Omega} g(t) \psi(t)
$$

for all $\psi \in W^{1,1}\left(0, T ; W^{1,1}(\Omega) \cap L^{\infty}(\Omega)\right) \cap L^{p}\left(0, T ; W^{1, p}(\Omega)\right)$ with $\psi(0)=\psi(T)=0$, and

$$
H(x, r)=\frac{1}{k} T_{k}(r-\hat{u}(x)+k \rho(x))
$$

where $\rho \in W^{1, p}(\Omega),-1 \leqslant \rho \leqslant 1$. Then, for any $\psi \in \mathcal{D}(\Omega), \psi \geqslant 0$, having in mind 40 , we have

$$
\begin{aligned}
\frac{\mathrm{d}}{\mathrm{d} t} \int_{\Omega}\left(\int _ { \hat { z } } ^ { z ( t ) } \frac { 1 } { k } T _ { k } \left(\left(\gamma^{-1}\right)^{0}(\tau)\right.\right. & -\hat{u}+k \rho) \mathrm{d} \tau) \psi \\
& +\int_{\Omega}(\mathbf{a}(x, D u(t))-\mathbf{a}(x, D \hat{u})) \cdot D\left(\frac{1}{k} T_{k}(u(t)-\hat{u}+k \rho) \psi\right) \\
& =\int_{\Omega}(f(t)-\hat{f}) \frac{1}{k} T_{k}(u(t)-\hat{u}+k \rho) \psi \quad \operatorname{in} \mathcal{D}^{\prime}(] 0, T[) .
\end{aligned}
$$

Now, it is easy to see that

$$
\begin{aligned}
& \left.\lim _{k \rightarrow 0} \int_{\hat{z}}^{z(t)} \frac{1}{k} T_{k}\left(\left(\gamma^{-1}\right)^{0}(\tau)-\hat{u}+k \rho\right)\right) \mathrm{d} \tau=\int_{\hat{z}}^{z(t)}\left[\operatorname{sign}_{0}\left(\left(\gamma^{-1}\right)^{0}(\tau)-\hat{u}\right)+\rho \chi_{\left\{\tau:\left(\gamma^{-1}\right)^{0}(\tau)=\hat{u}\right\}}\right] \mathrm{d} \tau \\
& \quad=\int_{\hat{z}}^{z(t)}\left[\operatorname{sign}_{0}(\tau-\hat{z})+\left(\rho-\operatorname{sign}_{0}(\tau-\hat{z})\right) \chi_{\left\{\tau:\left(\gamma^{-1}\right)^{0}(\tau)=\hat{u}\right\}}+\operatorname{sign}_{0}\left(\left(\gamma^{-1}\right)^{0}(\tau)-\hat{u}\right) \chi_{\{\tau=\hat{z}\}}\right] \mathrm{d} \tau \\
& \quad=\int_{\hat{z}}^{z(t)}\left[\operatorname{sign}_{0}(\tau-\hat{z})+\left(\rho-\operatorname{sign}_{0}(\tau-\hat{z})\right) \chi_{\left\{\tau:\left(\gamma^{-1}\right)^{0}(\tau)=\hat{u}\right\}}\right] \mathrm{d} \tau \\
& \quad=|z(t)-\hat{z}|+\int_{\hat{z}}^{z(t)}\left(\rho-\operatorname{sign}_{0}(\tau-\hat{z})\right) \chi_{\left\{\tau:\left(\gamma^{-1}\right)^{0}(\tau)=\hat{u}\right\}} .
\end{aligned}
$$

Hence, taking limits in (41) as $k$ goes to 0 , we get

$$
\begin{aligned}
\frac{\mathrm{d}}{\mathrm{d} t} \int_{\Omega}\left(|z(t)-\hat{z}|+\int_{\hat{z}}^{z(t)}\left(\rho-\operatorname{sign}_{0}(\tau-\hat{z})\right) \chi_{\left\{\tau:\left(\gamma^{-1}\right)^{0}(\tau)=\hat{u}\right\}}\right) \psi \\
+\int_{\Omega} \operatorname{sign}_{0}(u(t)-\hat{u})(\mathbf{a}(x, D u(t))-\mathbf{a}(x, D \hat{u})) \cdot D \psi
\end{aligned}
$$




$$
\begin{aligned}
\leqslant & \int_{\Omega}(f(t)-\hat{f})\left(\operatorname{sign}_{0}(z(t)-\hat{z})+\operatorname{sign}_{0}(u(t)-\hat{u}) \chi_{\{x \in \Omega: z(t)=\hat{z}\}}\right) \psi \\
& +\int_{\Omega}(f(t)-\hat{f})\left(\rho-\operatorname{sign}_{0}(z(t)-\hat{z})\right) \chi_{\{x \in \Omega: u(t)=\hat{u}\}} \psi
\end{aligned}
$$

and integrating between $\hat{t}, t \in] 0, T[$, we get

$$
\begin{aligned}
\int_{\Omega}|z(t)-\hat{z}| \psi- & \int_{\Omega}|z(\hat{t})-\hat{z}| \psi+\int_{\Omega} \int_{z(\hat{t})}^{z(t)}\left(\rho-\operatorname{sign}_{0}(\tau-\hat{z})\right) \chi_{\left\{\tau:\left(\gamma^{-1}\right)^{0}(\tau)=\hat{u}\right\}} \psi \\
& +\int_{\hat{t}}^{t} \int_{\Omega} \operatorname{sign}_{0}(u(\tau)-\hat{u})(\mathbf{a}(x, D u(\tau))-\mathbf{a}(x, D \hat{u})) \cdot D \psi \\
\leqslant & \int_{\hat{t}}^{t} \int_{\Omega}(f(\tau)-\hat{f})\left(\operatorname{sign}_{0}(z(\tau)-\hat{z})+\operatorname{sign}_{0}(u(\tau)-\hat{u}) \chi_{\{x \in \Omega: z(\tau)=\hat{z}\}}\right) \psi \\
& +\int_{\hat{t}}^{t} \int_{\Omega}(f(\tau)-\hat{f})\left(\rho-\operatorname{sign}_{0}(z(\tau)-\hat{z})\right) \chi_{\{x \in \Omega: u(\tau)=\hat{u}\}} \psi .
\end{aligned}
$$

Since in the last expression there are no space derivatives of $\rho$, we can take, for each $t$ fixed, $\rho=$ $\operatorname{sign}_{0}(z(t)-\hat{z})$. Then the second term in the above expression is positive and we have, for any $\hat{t}, t \in] 0, T[$,

$$
\begin{aligned}
\int_{\Omega}|z(t)-\hat{z}| \psi & -\int_{\Omega}|z(\hat{t})-\hat{z}| \psi+\int_{\hat{t}}^{t} \int_{\Omega} \operatorname{sign}_{0}(u(\tau)-\hat{u})(\mathbf{a}(x, D u(\tau))-\mathbf{a}(x, D \hat{u})) \cdot D \psi \\
\leqslant & \int_{\hat{t}}^{t} \int_{\Omega}(f(\tau)-\hat{f})\left(\operatorname{sign}_{0}(z(\tau)-\hat{z})+\operatorname{sign}_{0}(u(\tau)-\hat{u})\right) \chi_{\{x \in \Omega: z(\tau)=\hat{z}\}} \psi \\
& +\int_{\hat{t}}^{t} \int_{\Omega}(f(\tau)-\hat{f})\left(\operatorname{sign}_{0}(z(t)-\hat{z})-\operatorname{sign}_{0}(z(\tau)-\hat{z})\right) \chi_{\{x \in \Omega: u(\tau)=\hat{u}\}} \psi
\end{aligned}
$$

Let

$$
\begin{aligned}
\varphi_{1}(t):= & \int_{\Omega}|z(t)-\hat{z}| \psi \\
\varphi_{2}(\tau):= & -\int_{\Omega} \operatorname{sign}_{0}(u(\tau)-\hat{u})(\mathbf{a}(x, D u(\tau))-\mathbf{a}(x, D \hat{u})) D \psi \\
& +\int_{\Omega}(f(\tau)-\hat{f})\left(\operatorname{sign}_{0}(z(\tau)-\hat{z})+\operatorname{sign}_{0}(u(\tau)-\hat{u})\right) \chi_{\{x \in \Omega: z(\tau)(x)=\hat{z}(x)\}} \psi
\end{aligned}
$$

and

$$
\varphi_{3}(t, \tau):=\int_{\Omega}(f(\tau)-\hat{f})\left(\operatorname{sign}_{0}(z(t)-\hat{z})-\operatorname{sign}_{0}(z(\tau)-\hat{z})\right) \chi_{\{x \in \Omega: u(\tau)(x)=\hat{u}(x)\}} \psi .
$$

Then, taking in $42 \hat{t}=t-h, h>0$, dividing by $h$ and letting $h$ go to 0 , for any $\eta \in \mathcal{D}(] 0, T[)$, $\eta \geqslant 0$, we get 


$$
\begin{aligned}
& -\int_{0}^{T} \varphi_{1}(t) \eta_{t}(t) \mathrm{d} t=-\lim _{h \rightarrow 0^{+}} \int_{0}^{T} \varphi_{1}(t) \frac{\eta(t+h)-\eta(t)}{h} \mathrm{~d} t \\
& \quad=\lim _{h \rightarrow 0^{+}} \int_{0}^{T} \frac{\varphi_{1}(t)-\varphi_{1}(t-h)}{h} \eta(t) \mathrm{d} t \\
& \leqslant \lim _{h \rightarrow 0^{+}}\left(\int_{0}^{T} \frac{1}{h}\left(\int_{t-h}^{t} \varphi_{2}(\tau) \mathrm{d} \tau\right) \eta(t) \mathrm{d} t+\int_{0}^{T} \frac{1}{h}\left(\int_{t-h}^{t} \varphi_{3}(t, \tau) \mathrm{d} \tau\right) \eta(t) \mathrm{d} t\right) .
\end{aligned}
$$

Now, by the dominated convergence theorem,

$$
\begin{aligned}
\lim _{h \rightarrow 0^{+}} \int_{0}^{T} \frac{1}{h}\left(\int_{t-h}^{t} \varphi_{2}(\tau) \mathrm{d} \tau\right) \eta(t) \mathrm{d} t & =-\lim _{h \rightarrow 0^{+}} \int_{0}^{T}\left(\int_{0}^{t} \varphi_{2}(\tau) \mathrm{d} \tau\right) \frac{\eta(t+h)-\eta(t)}{h} \mathrm{~d} t \\
& =-\int_{0}^{T}\left(\int_{0}^{t} \varphi_{2}(\tau) \mathrm{d} \tau\right) \eta_{t}(t) \mathrm{d} t=\int_{0}^{T} \varphi_{2}(t) \eta(t) \mathrm{d} t
\end{aligned}
$$

On the other hand, for $h$ small enough,

$$
\int_{0}^{T} \frac{1}{h}\left(\int_{t-h}^{t} \varphi_{3}(t, \tau) \mathrm{d} \tau\right) \eta(t) \mathrm{d} t=\int_{0}^{T} \frac{1}{h}\left(\int_{\tau}^{\tau+h} \varphi_{3}(t, \tau) \eta(t) \mathrm{d} t\right) \mathrm{d} \tau
$$

Now,

$$
\begin{aligned}
& \left|\int_{0}^{T} \frac{1}{h}\left(\int_{\tau}^{\tau+h} \varphi_{3}(t, \tau) \eta(t) \mathrm{d} t\right) \mathrm{d} \tau\right| \\
& \leqslant \int_{0}^{T} \frac{1}{h}\left(\int_{\tau}^{\tau+h} \int_{\Omega}|f(\tau)-\hat{f}|\left|\operatorname{sign}_{0}(z(t)-\hat{z})-\operatorname{sign}_{0}(z(\tau)-\hat{z})\right| \eta(t) \psi(x) \mathrm{d} x \mathrm{~d} t\right) \mathrm{d} \tau \\
& \leqslant\|\psi\|_{L^{\infty}(\Omega)}\|\eta\|_{L^{\infty}(0, T)} \int_{0}^{T}\left[\int_{\Omega}|f(\tau)-\hat{f}| \mathrm{d} x\right. \\
& \left.\times \frac{1}{h} \int_{\tau}^{\tau+h}\left\|\operatorname{sign}_{0}(z(t)-\hat{z})-\operatorname{sign}_{0}(z(\tau)-\hat{z})\right\|_{L^{\infty}(\Omega)} \mathrm{d} t\right] \mathrm{d} \tau
\end{aligned}
$$

Moreover, for all Lebesgue points of the $L^{1}\left(0, T ; L^{\infty}(\Omega)\right)$-function $\operatorname{sign}_{0}(z(\cdot)-\hat{z})$, and so for a.e. $\tau \in] 0, T[$, we have

$$
\lim _{h \rightarrow 0^{+}} \frac{1}{h} \int_{\tau}^{\tau+h}\left\|\operatorname{sign}_{0}(z(t)-\hat{z})-\operatorname{sign}_{0}(z(\tau)-\hat{z})\right\|_{L^{\infty}(\Omega)} \mathrm{d} t=0 .
$$

Consequently, since

$$
\begin{aligned}
\left(\int_{\Omega}|f(\tau)-\hat{f}| \mathrm{d} x\right) \frac{1}{h} \int_{\tau}^{\tau+h}\left\|\operatorname{sign}_{0}(z(t)-\hat{z})-\operatorname{sign}_{0}(z(\tau)-\hat{z})\right\|_{L^{\infty}(\Omega)} \mathrm{d} t & \\
& \leqslant 2 \int_{\Omega}|f(\tau)-\hat{f}| \mathrm{d} x
\end{aligned}
$$


which is a function in $L^{1}(0, T)$, by the dominated convergence theorem, we get

$$
\lim _{h \rightarrow 0^{+}} \int_{0}^{T} \frac{1}{h}\left(\int_{t-h}^{t} \varphi_{3}(t, \tau) \mathrm{d} \tau\right) \eta(t) \mathrm{d} t=\lim _{h \rightarrow 0^{+}} \int_{0}^{T} \frac{1}{h}\left(\int_{\tau}^{\tau+h} \varphi_{3}(t, \tau) \eta(t) \mathrm{d} t\right) \mathrm{d} \tau=0 .
$$

Therefore, from 43 we obtain

$$
\begin{aligned}
& \frac{\mathrm{d}}{\mathrm{d} t} \int_{\Omega}|z(t)-\hat{z}| \psi+\int_{\Omega} \operatorname{sign}_{0}(u(t)-\hat{u})(\mathbf{a}(x, D u(t)-\mathbf{a}(x, D \hat{u})) \cdot D \psi \\
& \quad \leqslant \int_{\Omega}(f(t)-\hat{f})\left(\operatorname{sign}_{0}(z(t)-\hat{z})+\operatorname{sign}_{0}(u(t)-\hat{u}) \chi_{\{x \in \Omega: z(t)=\hat{z}\}}\right) \psi
\end{aligned}
$$

in $\mathcal{D}^{\prime}(] 0, T[)$.

THEOREM 5.3 Let $(z, w)$ be a weak solution of $P_{\gamma, \beta}\left(f, g, z_{0}, w_{0}\right)$ in $[0, T]$. Let $(\hat{f}, \hat{g}) \in$ $\mathcal{B}^{\gamma, \beta}(\hat{z}, \hat{w})$. Then

$$
\begin{aligned}
\frac{\mathrm{d}}{\mathrm{d} t} \int_{\Omega}|z(t)-\hat{z}|+\frac{\mathrm{d}}{\mathrm{d} t} \int_{\partial \Omega} & |w(t)-\hat{w}| \\
\leqslant & \int_{\Omega}(f(t)-\hat{f}) \operatorname{sign}_{0}(z(t)-\hat{z})+\int_{\{x \in \Omega: z(t)=\hat{z}\}}|f(t)-\hat{f}| \\
& +\int_{\partial \Omega}(g(t)-\hat{g}) \operatorname{sign}_{0}(w(t)-\hat{w})+\int_{\{x \in \partial \Omega: w(t)=\hat{w}\}}|g(t)-\hat{g}|
\end{aligned}
$$

in $\mathcal{D}^{\prime}(] 0, T[)$, that is, since $(z(0), w(0))=\left(z_{0}, w_{0}\right),(z, w)$ is an integral solution of 10$]$ in $[0, T]$.

Proof. Let $u \in L^{p}\left(0, T ; W^{1, p}(\Omega)\right)$ be such that $z \in \gamma(u)$ a.e. in $Q_{T}$ and $w \in \beta(u)$ a.e. on $S_{T}$ as in Definition 2.2. and let $\hat{u} \in W^{1, p}(\Omega)$ be such that $\hat{z} \in \gamma(\hat{u})$ a.e. in $\Omega$ and $\hat{w} \in \gamma(\hat{u})$ a.e. in $\partial \Omega$ as in the definition of $\mathcal{B}^{\gamma, \beta}$.

Thanks to Lemma 5.2, for any $\psi \in \mathcal{D}(\Omega), 0 \leqslant \psi \leqslant 1$, we have

$$
\begin{aligned}
\frac{\mathrm{d}}{\mathrm{d} t} \int_{\Omega}|z(t)-\hat{z}| \psi+ & \int_{\Omega} \operatorname{sign}_{0}(u(t)-\hat{u})(\mathbf{a}(x, D u(t)-\mathbf{a}(x, D \hat{u})) \cdot D \psi \\
& \leqslant \int_{\Omega}(f(t)-\hat{f}) \operatorname{sign}_{0}(z(t)-\hat{z}) \psi+\int_{\{x \in \Omega: z(t)=\hat{z}\}}|f(t)-\hat{f}| \psi
\end{aligned}
$$

in $\mathcal{D}^{\prime}(] 0, T[)$. Now, for the second term on the left hand side we have

$$
\begin{aligned}
\int_{\Omega} \operatorname{sign}_{0}(u(t) & -\hat{u})(\mathbf{a}(x, D u(t)-\mathbf{a}(x, D \hat{u})) \cdot D \psi \\
& =\int_{\Omega} \operatorname{sign}_{0}(u(t)-\hat{u})(\mathbf{a}(x, D u(t)-\mathbf{a}(x, D \hat{u})) \cdot D(\psi-1) \\
& \geqslant \lim _{k \rightarrow 0} \int_{\Omega}(\mathbf{a}(x, D u(t))-\mathbf{a}(x, D \hat{u})) \cdot D\left(\frac{1}{k} T_{k}(u(t)-\hat{u}+k \rho)(\psi-1)\right),
\end{aligned}
$$


where $\rho \in W^{1, p}(\Omega),-1 \leqslant \rho \leqslant 1$. Using again Lemma 4.1 we get

$$
\begin{aligned}
\int_{\Omega}(\mathbf{a}(x, D u(t)-\mathbf{a}(x, D \hat{u})) & \cdot D\left(\frac{1}{k} T_{k}(u(t)-\hat{u}+k \rho)(\psi-1)\right) \\
= & -\frac{\mathrm{d}}{\mathrm{d} t} \int_{\Omega}\left(\int_{\hat{z}}^{z(t)} \frac{1}{k} T_{k}\left(\left(\gamma^{-1}\right)^{0}(s)-\hat{u}+k \rho\right) \mathrm{d} s\right)(\psi-1) \\
& +\frac{\mathrm{d}}{\mathrm{d} t} \int_{\partial \Omega}\left(\int_{\hat{w}}^{w(t)} \frac{1}{k} T_{k}\left(\left(\beta^{-1}\right)^{0}(s)-\hat{u}+k \rho\right) \mathrm{d} s\right) \\
& +\int_{\Omega}(f(t)-\hat{f}) \frac{1}{k} T_{k}(u(t)-\hat{u}+k \rho)(\psi-1) \\
& -\int_{\partial \Omega}(g(t)-\hat{g}) \frac{1}{k} T_{k}(u(t)-\hat{u}+k \rho),
\end{aligned}
$$

which converges as $k$ goes to 0 to

$$
\begin{aligned}
& -\frac{\mathrm{d}}{\mathrm{d} t} \int_{\Omega}\left(|z(t)-\hat{z}|-\int_{\hat{z}}^{z(t)}\left(\rho-\operatorname{sign}_{0}(s-\hat{z})\right) \chi_{\left\{s:\left(\gamma^{-1}\right)^{0}(s)=\hat{u}\right\}}\right)(\psi-1) \\
& +\frac{\mathrm{d}}{\mathrm{d} t} \int_{\partial \Omega}\left(|w(t)-\hat{w}|+\int_{\hat{w}}^{w(t)}\left(\rho-\operatorname{sign}_{0}(s-\hat{w})\right) \chi_{\left\{s:\left(\beta^{-1}\right)^{0}(s)=\hat{u}\right\}}\right) \\
& +\int_{\Omega}(f(t)-\hat{f})\left(\operatorname{sign}_{0}(z(t)-\hat{z})+\operatorname{sign}_{0}(u(t)-\hat{u}) \chi_{\{x \in \Omega: z(t)=\hat{z}\}}\right)(\psi-1) \\
& +\int_{\Omega}(f(t)-\hat{f})\left(\rho-\operatorname{sign}_{0}(z(t)-\hat{z})\right) \chi_{\{x \in \Omega: u(t)=\hat{u}\}}(\psi-1) \\
& -\int_{\partial \Omega}(g(t)-\hat{g})\left(\operatorname{sign}_{0}(w(t)-\hat{w})+\operatorname{sign}_{0}(u(t)-\hat{u}) \chi_{\{x \in \partial \Omega: w(t)=\hat{w}\}}\right) \\
& -\int_{\partial \Omega}(g(t)-\hat{g})\left(\rho-\operatorname{sign}_{0}(w(t)-\hat{w})\right) \chi_{\{x \in \partial \Omega: u(t)=\hat{u}\}} .
\end{aligned}
$$

Therefore, taking into account (45) and (46) in (44), replacing $\psi$ by $\psi_{n}$ such that $L^{1}(\Omega)-\lim _{n} \psi_{n}=1$, and taking limits as $n$ goes to $+\infty$ we obtain

$$
\begin{aligned}
\frac{\mathrm{d}}{\mathrm{d} t} \int_{\Omega}|z(t)-\hat{z}|+ & \frac{\mathrm{d}}{\mathrm{d} t} \int_{\partial \Omega}\left(|w(t)-\hat{w}|+\int_{\hat{w}}^{w(t)}\left(\rho-\operatorname{sign}_{0}(s-\hat{w})\right) \chi_{\left\{s:\left(\beta^{-1}\right)^{0}(s)=\hat{u}\right\}}\right) \\
\leqslant & \int_{\Omega}(f(t)-\hat{f}) \operatorname{sign}_{0}(z(t)-\hat{z})+\int_{\{x \in \Omega: z(t)=\hat{z}\}}|f(t)-\hat{f}| \\
& +\int_{\partial \Omega}(g(t)-\hat{g})\left(\operatorname{sign}_{0}(w(t)-\hat{w})+\operatorname{sign}_{0}(u(t)-\hat{u}) \chi_{\{x \in \partial \Omega: w(t)=\hat{w}\}}\right) \\
& +\int_{\partial \Omega}(g(t)-\hat{g})\left(\rho-\operatorname{sign}_{0}(w(t)-\hat{w})\right) \chi_{\{x \in \partial \Omega: u(t)=\hat{u}\} .}
\end{aligned}
$$

Finally, by a similar argument to the one used in Lemma 5.2, we finish the proof.

REMARK 5.4 It is easy to see that Theorem 2.5 also holds for data $\left(z_{0}, w_{0}\right) \in V^{1, p}(\Omega) \times$ $V^{1, p}(\partial \Omega)$ and $(f, g) \in L^{p^{\prime}}\left(0, T ; V^{1, p}(\Omega)\right) \times L^{p^{\prime}}\left(0, T ; V^{1, p}(\partial \Omega)\right)$ satisfying conditions (4)-(6), in particular, if $p>N$, for $\left(z_{0}, w_{0}\right) \in L^{1}(\Omega) \times L^{1}(\partial \Omega)$ and $(f, g) \in L^{1}\left(0, T ; L^{1}(\Omega)\right) \times$ $L^{1}\left(0, T ; L^{1}(\partial \Omega)\right)$ satisfying $(4)-(6)$. 


\section{Appendix}

This appendix is devoted to the proof of Theorem 3.5. For that we need some lemmas.

LEMma 6.1 Assume $\gamma, \beta: \mathbb{R} \rightarrow \mathbb{R}$ are strictly increasing functions with $\operatorname{Ran}(\gamma)=\operatorname{Ran}(\beta)=\mathbb{R}$. Let $\phi \in L^{\infty}(\Omega)$ and $\psi \in L^{\infty}(\partial \Omega)$. Then, if $[u, z, w]$ is a weak solution of problem $\left(S_{\phi, \psi}^{\gamma, \beta}\right)$, we have

$$
\inf \left\{\gamma^{-1}(\inf \phi), \beta^{-1}(\inf \psi)\right\} \leqslant u \leqslant \max \left\{\gamma^{-1}(\sup \phi), \beta^{-1}(\sup \psi)\right\} .
$$

Proof. By Theorem 3.2(ii), if

$$
a:=\inf \left\{\gamma^{-1}(\inf \phi), \beta^{-1}(\inf \psi)\right\}, \quad b:=\max \left\{\gamma^{-1}(\sup \phi), \beta^{-1}(\sup \psi)\right\},
$$

we have

$$
\begin{aligned}
& \int_{\Omega}(\gamma(a)-z)^{+}+\int_{\partial \Omega}(\beta(a)-w)^{+} \leqslant \int_{\Omega}(\gamma(a)-\phi)^{+}+\int_{\partial \Omega}(\beta(a)-\psi)^{+}, \\
& \int_{\Omega}(z-\gamma(b))^{+}+\int_{\partial \Omega}(w-\beta(b))^{+} \leqslant \int_{\Omega}(\phi-\gamma(b))^{+}+\int_{\partial \Omega}(\psi-\beta(b))^{+},
\end{aligned}
$$

and hence the result follows.

Now, for $m, n, r, l \in \mathbb{N}$, let

$$
\gamma_{l}^{m, n}(s)=\gamma_{l}(s)+\frac{1}{l}|s|^{p-2} s+\frac{1}{m} s^{+}-\frac{1}{n} s^{-}, \quad \beta_{r}^{m, n}(s)=\beta_{r}(s)+\frac{1}{m} s^{+}-\frac{1}{n} s^{-},
$$

where $\gamma_{l}$ and $\beta_{r}$ are the Yosida approximations of $\gamma$ and $\beta$ respectively. Then, by the above lemma, if $\left[u_{r, l}^{m, n}, z_{r, l}^{m, n}, w_{r, l}^{m, n}\right]$ is a weak solution of $\left(S_{\phi, \psi}^{\gamma_{l}^{m, n}, \beta_{r}^{m, n}}\right)$ for $\phi \in L^{\infty}(\Omega)$ and $\psi \in L^{\infty}(\partial \Omega)$, then

$$
\inf \left\{\left(\gamma_{r, l}^{m, n}\right)^{-1}(\inf \phi),\left(\beta_{r, l}^{m, n}\right)^{-1}(\inf \psi)\right\} \leqslant u_{r, l}^{m, n} \leqslant \sup \left\{\left(\gamma_{l}^{m, n}\right)^{-1}(\sup \phi),\left(\beta_{r}^{m, n}\right)^{-1}(\sup \psi)\right\} .
$$

Since

$$
\begin{aligned}
& \gamma^{m, n}(s):=\left(\liminf _{l \rightarrow+\infty} \gamma_{l}^{m, n}\right)(s)=\gamma(s)+\frac{1}{m} s^{+}-\frac{1}{n} s^{-}, \\
& \beta^{m, n}(s):=\left(\liminf _{r \rightarrow+\infty} \beta_{r}^{m, n}\right)(s)=\beta(s)+\frac{1}{m} s^{+}-\frac{1}{n} s^{-},
\end{aligned}
$$

the next lemma follows.

LEMMA 6.2 Assume $\lim _{l} \lim _{r} u_{r, l}^{m, n}=u^{m, n}$ a.e. in $\Omega$ or $\lim _{r} u_{r, r}^{m, n}=u^{m, n}$ a.e. in $\Omega$. Let $\phi \in$ $L^{\infty}(\Omega)$ and $\psi \in L^{\infty}(\partial \Omega)$. Then

$$
\begin{aligned}
\inf \left\{\inf \left(\gamma^{m, n}\right)^{-1}(\inf \phi), \inf \left(\beta^{m, n}\right)^{-1}(\inf \psi)\right\} & \leqslant u^{m, n} \\
& \leqslant \sup \left\{\sup \left(\gamma^{m, n}\right)^{-1}(\sup \phi), \sup \left(\beta^{m, n}\right)^{-1}(\sup \psi)\right\} .
\end{aligned}
$$

Let $n(m)$ be a subsequence in $\mathbb{N}$. Since

$$
\liminf _{m \rightarrow \infty} \gamma^{m, n(m)}=\gamma, \quad \liminf _{m \rightarrow \infty} \beta^{m, n(m)}=\beta,
$$

the following result holds. 
LEMMA 6.3 Assume $\lim _{m \rightarrow \infty} u^{m, n(m)}=u$ a.e. in $\Omega$. If $a_{0} \leqslant \phi \leqslant a_{1}$ and $b_{0} \leqslant \psi \leqslant b_{1}$, where

- $\gamma_{-}<a_{0}<0$ if $\gamma_{-}<0$ and $0 \leqslant a_{0}$ if $\gamma_{-}=0$,

- $0<a_{1}<\gamma_{+}$if $\gamma_{+}>0$ and $a_{1} \leqslant 0$ if $\gamma_{+}=0$,

- $\beta_{-}<b_{0}<0$ if $\beta_{-}<0$ and $0 \leqslant b_{0}$ if $\beta_{-}=0$,

- $0<b_{1}<\beta_{+}$if $\beta_{+}>0$ and $b_{1} \leqslant 0$ if $\beta_{+}=0$,

then

$$
\inf \left\{A_{0}, B_{0}\right\} \leqslant u \leqslant \sup \left\{A_{1}, B_{1}\right\}
$$

where $A_{0}=\inf \gamma^{-1}\left(a_{0}\right)$ if $\gamma_{-}<0, A_{0}=0$ if $\gamma_{-}=0, B_{0}=\inf \beta^{-1}\left(b_{0}\right)$ if $\beta_{-}<0, B_{0}=0$ if $\beta_{-}=0, A_{1}=\sup \gamma^{-1}\left(a_{1}\right)$ if $\gamma_{+}>0, A_{1}=0$ if $\gamma_{+}=0, B_{1}=\sup \beta^{-1}\left(b_{1}\right)$ if $\beta_{+}>0$ and $B_{1}=0$ if $\beta_{+}=0$.

Proof of Theorem 3.5. It is obvious that

$$
{\overline{D\left(\mathcal{B}^{\gamma, \beta}\right)}}^{L^{1}(\Omega) \times L^{1}(\partial \Omega)} \subset\left\{(z, w) \in L^{1}(\Omega) \times L^{1}(\partial \Omega): \gamma_{-} \leqslant z \leqslant \gamma_{+}, \beta_{-} \leqslant w \leqslant \beta_{+}\right\}
$$

To obtain the other inclusion, it is enough to take $(z, w) \in L^{\infty}(\Omega) \times L^{\infty}(\partial \Omega)$ with $a_{0} \leqslant z \leqslant a_{1}$ and $b_{0} \leqslant w \leqslant b_{1}$, where the constants $a_{i}, b_{i}, i=0,1$, satisfy

- $\gamma_{-}<a_{0}<0$ if $\gamma_{-}<0$ and $a_{0}=0$ if $\gamma_{-}=0$,

- $0<a_{1}<\gamma_{+}$if $\gamma_{+}>0$ and $a_{1}=0$ if $\gamma_{+}=0$,

- $\beta_{-}<b_{0}<0$ if $\beta_{-}<0$ and $b_{0}=0$ if $\beta_{-}=0$,

- $0<b_{1}<\beta_{+}$if $\beta_{+}>0$ and $b_{1}=0$ if $\beta_{+}=0$,

and to prove that $(z, w) \in{\overline{D\left(\mathcal{B}^{\gamma, \beta}\right)}}^{X}$.

Given $(z, w) \in L^{\infty}(\Omega) \times L^{\infty}(\partial \Omega)$ with $a_{0} \leqslant z \leqslant a_{1}$ and $b_{0} \leqslant w \leqslant b_{1}$, we set

$$
\left(z_{n}, w_{n}\right)=\left(I+\frac{1}{n} \mathcal{B}^{\gamma, \beta}\right)^{-1}(z, w), \quad n \in \mathbb{N}
$$

Let us see that there exists a subsequence, denoted $\left(z_{n}, w_{n}\right)$ again, such that

$$
\left(z_{n}, w_{n}\right) \rightarrow(z, w) \quad \text { in } L^{1}(\Omega) \times L^{1}(\partial \Omega),
$$

which implies that $(z, w) \in{\overline{D\left(\mathcal{B}^{\gamma}, \beta\right)}}^{X}$

Since $\left(\left(z_{n}, w_{n}\right), n\left(z-z_{n}, w-w_{n}\right)\right) \in \mathcal{B}^{\gamma, \beta}$, there exist $u_{n} \in W^{1, p}(\Omega)$ such that $\left[u_{n}, z_{n}, w_{n}\right]$ is a weak solution of problem $\left(S_{z_{n}+n\left(z-z_{n}\right), w_{n}+n\left(w-w_{n}\right)}^{\gamma, \beta}\right.$. Hence, $z_{n}(x) \in \gamma\left(u_{n}(x)\right)$ a.e. in $\Omega, w_{n}(x) \in$ $\beta\left(u_{n}(x)\right)$ a.e. in $\partial \Omega$ and

$$
\frac{1}{n} \int_{\Omega} \mathbf{a}\left(x, D u_{n}\right) \cdot D \phi+\int_{\Omega} z_{n} \phi+\int_{\partial \Omega} w_{n} \phi=\int_{\Omega} z \phi+\int_{\partial \Omega} w \phi
$$

for all $\phi \in W^{1, p}(\Omega)$. 
Note that if $\mathbf{a}_{n}(x, \xi):=\frac{1}{n} \mathbf{a}(x, \xi)$, then $\left[u_{n}, z_{n}, w_{n}\right]$ is a weak solution of the problem

$$
\left(\mathbf{a}_{n} S_{z, w}^{\gamma, \beta}\right) \quad \begin{cases}-\operatorname{div} \mathbf{a}_{n}(x, D u)+\gamma(u) \ni z & \text { in } \Omega \\ \mathbf{a}_{n}(x, D u) \cdot \eta+\beta(u) \ni w & \text { on } \partial \Omega\end{cases}
$$

and by uniqueness, we can assume that $\left[u_{n}, z_{n}, w_{n}\right]$ is the weak solution of problem $\left(a_{n} S_{z, w}^{\gamma, \beta}\right)$ given in Theorem 3.3. This construction is done as follows (see [6]). Firstly, we find a weak solution $\left[\left(u_{n}\right)_{r}^{m, k},\left(z_{n}\right)_{r}^{m, k},\left(w_{n}\right)_{r}^{m, k}\right]$ of $\left(\mathbf{a}_{n} S_{z, w}^{\gamma_{r}^{m, k}, \beta_{r}^{m, k}}\right)$ in case $\operatorname{Dom}(\beta)=\mathbb{R}$, and $\left[\left(u_{n}\right)_{r, l}^{m, k},\left(z_{n}\right)_{r, l}^{m, k},\left(w_{n}\right)_{r, l}^{m, k}\right]$ of $\left(\mathbf{a}_{n} S_{z, w}^{\gamma_{l}^{m, k}, \beta_{r}^{m, k}}\right)$ in case a is smooth. If $\operatorname{Dom}(\beta)=\mathbb{R}$, taking limits as $r$ goes to $+\infty$, we have

$$
\begin{aligned}
\lim _{r}\left(u_{n}\right)_{r}^{m, k} & =\left(u_{n}\right)^{m, k} & & \text { in } L^{1}(\Omega), \\
\lim _{r}\left(z_{n}\right)_{r}^{m, k} & =\left(z_{n}\right)^{m, k} & & \text { weakly in } L^{1}(\Omega), \\
\lim _{r}\left(w_{n}\right)_{r}^{m, k} & =\left(w_{n}\right)^{m, k} & & \text { weakly in } L^{1}(\partial \Omega),
\end{aligned}
$$

$\left[\left(u_{n}\right)^{m, k},\left(z_{n}\right)^{m, k},\left(w_{n}\right)^{m, k}\right]$ being a weak solution of $\left(S_{z, w}^{\gamma^{m, k}, \beta^{m, k}}\right)$; in case a is smooth, taking limits as $l$ goes to $+\infty$ we get

$$
\begin{aligned}
\lim _{l}\left(u_{n}\right)_{r, l}^{m, k}=\left(u_{n}\right)_{r}^{m, k} & \text { in } L^{1}(\Omega), \\
\lim _{l}\left(z_{n}\right)_{r, l}^{m, k}=\left(z_{n}\right)_{r}^{m, k} & \text { weakly in } L^{1}(\Omega), \\
\lim _{l}\left(w_{n}\right)_{r, l}^{m, k}=\left(w_{n}\right)_{r}^{m, k} & \text { weakly in } L^{1}(\partial \Omega),
\end{aligned}
$$

$\left[\left(u_{n}\right)_{r}^{m, k},\left(z_{n}\right)_{r}^{m, k},\left(w_{n}\right)_{r}^{m, k}\right]$ being a weak solution of $\left(S_{z, w}^{\gamma^{m, k}, \beta_{r}^{m, k}}\right)$, and taking limits as $r$ goes to $+\infty$, we obtain

$$
\begin{aligned}
& \lim _{r}\left(u_{n}\right)_{r}^{m, k}=\left(u_{n}\right)^{m, k} \quad \text { in } L^{1}(\Omega), \\
& \lim _{r}^{r}\left(z_{n}\right)_{r}^{m, k}=\left(z_{n}\right)^{m, k} \quad \text { weakly in } L^{1}(\Omega), \\
& \lim _{r}^{r}\left(w_{n}\right)_{r}^{m, k}=\left(w_{n}\right)^{m, k} \quad \text { weakly in } L^{1}(\partial \Omega),
\end{aligned}
$$

$\left[\left(u_{n}\right)^{m, k},\left(z_{n}\right)^{m, k},\left(w_{n}\right)^{m, k}\right]$ being a weak solution of $\left(S_{z, w}^{\gamma^{m, k}, \beta^{m, k}}\right)$. Moreover, in the case of a smooth,

$$
\left(w_{n}\right)^{m, k} \ll w-\mathbf{a}_{n}\left(x, D\left(\hat{u}_{n}\right)^{m, k}\right) \cdot \eta,
$$

$\left[\left(\hat{u}_{n}\right)^{m, k},\left(\hat{z}_{n}\right)^{m, k}\right]$ being a weak solution of

$$
\begin{cases}-\operatorname{div} \mathbf{a}_{n}\left(x, D\left(\hat{u}_{n}\right)^{m, k}\right)+\gamma\left(\left(\hat{u}_{n}\right)^{m, k}\right)+\frac{1}{m}\left(\left(\hat{u}_{n}\right)^{m, k}\right)^{+}-\frac{1}{k}\left(\left(\hat{u}_{n}\right)^{m, k}\right)^{-} \ni z & \text { in } \Omega, \\ \left(\hat{u}_{n}\right)^{m, k}=0 & \text { on } \partial \Omega .\end{cases}
$$

Finally, passing to the limit in $m$ for a suitable subsequence $\{k(m)\}$ in $\mathbb{N}$, we have

$$
\begin{aligned}
& \lim _{m \rightarrow \infty}\left(u_{n}\right)^{m, k(m)}=u_{n} \quad \text { in } L^{1}(\Omega), \\
& \lim _{m \rightarrow \infty}\left(z_{n}\right)^{m, k(m)}=z_{n} \quad \text { in } L^{1}(\Omega), \\
& \lim _{m \rightarrow \infty}\left(w_{n}\right)^{m, k(m)}=w_{n} \quad \text { in } L^{1}(\partial \Omega) \text {. }
\end{aligned}
$$


474

F. ANDRE ET AL.

If $\mathbf{a}$ is smooth,

$$
\begin{aligned}
\lim _{m \rightarrow \infty}\left(\hat{u}_{n}\right)^{m, k(m)} & =\hat{u}_{n} & & \text { in } L^{1}(\Omega), \\
\lim _{m \rightarrow \infty}\left(\hat{z}_{n}\right)^{m, k(m)} & =\hat{z}_{n} & & \text { in } L^{1}(\Omega), \\
\lim _{m \rightarrow \infty} \mathbf{a}_{n}\left(x, D\left(\hat{u}_{n}\right)^{m, k(m)}\right) \cdot \eta & =\mathbf{a}_{n}\left(x, D \hat{u}_{n}\right) \cdot \eta & & \text { in } L^{1}(\partial \Omega),
\end{aligned}
$$

$\left[\hat{u}_{n}, \hat{z}_{n}\right]$ being a weak solution of

$$
\begin{cases}-\operatorname{div} \mathbf{a}_{n}\left(x, D \hat{u}_{n}\right)+\gamma\left(\hat{u}_{n}\right) \ni z & \text { in } \Omega, \\ \hat{u}_{n}=0 & \text { on } \partial \Omega .\end{cases}
$$

Moreover (see [6]),

$$
\begin{gathered}
\hat{z}_{n} \ll z, \\
w_{n} \ll w-\mathbf{a}_{n}\left(x, D \hat{u}_{n}\right) \cdot \eta,
\end{gathered}
$$

and

$$
\int_{\partial \Omega}\left|\mathbf{a}_{n}\left(x, D \hat{u}_{n}\right) \cdot \eta\right| \leqslant \int_{\Omega}\left|z-\hat{z}_{n}\right| .
$$

Observe that, by Lemmas 6.1 6.3 $\left\{u_{n}\right\}$ is uniformly bounded in $L^{\infty}(\Omega)$; similarly, $\left\{\hat{u}_{n}\right\}$ is uniformly bounded in $L^{\infty}(\Omega)$. Therefore, since $\operatorname{Dom}(\gamma)=\mathbb{R},\left\{z_{n}\right\}$ and $\left\{\hat{z}_{n}\right\}$ are uniformly bounded in $L^{\infty}(\Omega)$, so there exists a subsequence (not relabelled) such that $z_{n}$ and $\hat{z}_{n}$ are weakly convergent in $L^{1}(\Omega)$. Also, in the case $\operatorname{Dom}(\beta)=\mathbb{R}$, there exists a subsequence (not relabelled) such that $w_{n}$ is weakly convergent in $L^{1}(\partial \Omega)$.

We now claim that

$$
\lim _{n \rightarrow \infty} \int_{\Omega} z_{n} \phi=\int_{\Omega} z \phi \quad \text { for every } \phi \in \mathcal{D}(\Omega) .
$$

Taking $\phi=u_{n}$ in (47), since $z_{n}(x) \in \gamma\left(u_{n}(x)\right)$ ae. in $\Omega, w_{n}(x) \in \beta\left(u_{n}(x)\right)$ a.e. in $\partial \Omega$, and $\left\{u_{n}\right\}$ is bounded in $L^{\infty}(\Omega)$, we get

$$
\int_{\Omega} \mathbf{a}\left(x, D u_{n}\right) \cdot D u_{n} \leqslant n\left(\int_{\Omega} z u_{n}+\int_{\partial \Omega} w u_{n}\right) \leqslant n C .
$$

Now, using $\left(\mathrm{H}_{1}\right)$ and $\left(\mathrm{H}_{2}\right)$, we have

$$
\begin{aligned}
\left(\int_{\Omega}\left|\mathbf{a}\left(x, D u_{n}\right)\right|^{p^{\prime}}\right)^{1 / p^{\prime}} & \leqslant \sigma\left(\int_{\Omega}\left(\varrho(x)+\left|D u_{n}\right|^{p-1}\right)^{p^{\prime}}\right)^{1 / p^{\prime}} \\
& \leqslant \sigma\left(\left(\int_{\Omega} \varrho(x)^{p^{\prime}}\right)^{1 / p^{\prime}}+\left(\int_{\Omega}\left|D u_{n}\right|^{p}\right)^{1 / p^{\prime}}\right) \\
& \leqslant \sigma\left(\left(\int_{\Omega} \varrho(x)^{p^{\prime}}\right)^{1 / p^{\prime}}+\left(\frac{1}{\lambda} \int_{\Omega} \mathbf{a}\left(x, D u_{n}\right) \cdot D u_{n}\right)^{1 / p^{\prime}}\right) \\
& \leqslant \sigma\|\|_{L^{p^{\prime}}(\Omega)}+\sigma\left(\frac{C}{\lambda} n\right)^{1 / p^{\prime}} .
\end{aligned}
$$


Consequently,

$$
\left(\int_{\Omega}\left|\frac{1}{n} \mathbf{a}\left(x, D u_{n}\right)\right|^{p^{\prime}}\right)^{1 / p^{\prime}} \leqslant \frac{\sigma\|\varrho\|_{L^{p^{\prime}}(\Omega)}}{n}+\sigma\left(\frac{C / \lambda}{n^{p^{\prime}-1}}\right)^{1 / p^{\prime}} .
$$

On the other hand, taking $\phi \in \mathcal{D}(\Omega)$ in 47) we have

$$
\frac{1}{n} \int_{\Omega} \mathbf{a}\left(x, D u_{n}\right) \cdot D \phi+\int_{\Omega} z_{n} \phi=\int_{\Omega} z \phi
$$

By (53), we get 52). Consequently,

$$
z_{n} \rightarrow z \quad \text { weakly in } L^{1}(\Omega) .
$$

Having in mind (54) and (53), it follows from (47) that

$$
\int_{\partial \Omega} w_{n} \phi \rightarrow \int_{\partial \Omega} w \phi \quad \text { for any } \phi \in W^{1, p}(\Omega) \cap L^{\infty}(\Omega) .
$$

Therefore, in the case $\operatorname{Dom}(\beta)=\mathbb{R}$, by 55 we find that

$$
w_{n} \rightarrow w \text { weakly in } L^{1}(\partial \Omega) .
$$

Similarly, $\hat{z}_{n} \rightarrow z$ weakly in $L^{1}(\Omega)$, hence by $49, \hat{z}_{n} \rightarrow z$ in $L^{1}(\Omega)$. Therefore, in the case of a smooth, from (50), 51) and a similar argument, we infer that

$$
w_{n} \rightarrow w \quad \text { weakly in } L^{1}(\partial \Omega) .
$$

Observe that for any $b \geqslant 0$ and $c \geqslant 0$, we also have

$$
\left(z_{n}-b\right)^{+} \rightarrow z_{b} \geqslant(z-b)^{+}, \quad\left(w_{n}-c\right)^{+} \rightarrow w_{c} \geqslant(w-c)^{+} .
$$

Now, if $c \notin \operatorname{Ran}(\beta)$, then $\int_{\partial \Omega}\left(w_{n}-c\right)^{+} \leqslant 0$, therefore $\int_{\partial \Omega}(w-c)^{+} \leqslant \int_{\partial \Omega} w_{c} \leqslant 0$, and so

$$
w_{c}=(w-c)^{+}
$$

On the other hand, if $c \in \operatorname{Ran}(\beta)$, there exists $a \geqslant 0$ such that $c \in \beta(a)$; taking $b \in \gamma(a)$, since $[a, b, c]$ is an entropy solution of problem $\left(\mathbf{a}_{n} S_{b, c}^{\gamma, \beta}\right)$, we have

$$
\int_{\Omega}\left(z_{n}-b\right)^{+}+\int_{\partial \Omega}\left(w_{n}-c\right)^{+} \leqslant \int_{\Omega}(z-b)^{+}+\int_{\partial \Omega}(w-c)^{+} .
$$

Taking limits in 56, we get

$$
\int_{\Omega}(z-b)^{+}+\int_{\partial \Omega}(w-c)^{+} \leqslant \int_{\Omega} z_{b}+\int_{\partial \Omega} w_{c} \leqslant \int_{\Omega}(z-b)^{+}+\int_{\partial \Omega}(w-c)^{+},
$$

hence again

$$
w_{c}=(w-c)^{+}
$$


Consequently, for any $c \geqslant 0$,

$$
\left(w_{n}-c\right)^{+} \rightarrow(w-c)^{+} \quad \text { weakly in } L^{1}(\partial \Omega) .
$$

Working similarly, we also get

$$
\left(w_{n}+c\right)^{-} \rightarrow(w+c)^{-} \text {weakly in } L^{1}(\partial \Omega) .
$$

By (57) and 58), proceeding as in the proof of [13, Proposition 2.11], we conclude that

$$
w_{n} \rightarrow w \text { in } L^{1}(\partial \Omega)
$$

For $b \geqslant 0$, we have

$$
\left(z_{n}-b\right)^{+} \rightarrow z_{b} \geqslant(z-b)^{+}
$$

Now, if $b \notin \operatorname{Ran}(\gamma)$, that $\int_{\Omega}(z-b)^{+} \leqslant \int_{\Omega} z_{b} \leqslant 0$, hence $z_{b}=(z-b)^{+}$. On the other hand, if $b \in \operatorname{Ran}(\gamma)$, then there exists $a \geqslant 0$ such that $b \in \gamma(a)$. If $a \in \operatorname{Dom}(\beta)$, taking $c \in \beta(a)$, we obtain

$$
\int_{\Omega}\left(z_{n}-b\right)^{+}+\int_{\partial \Omega}\left(w_{n}-c\right)^{+} \leqslant \int_{\Omega}(z-b)^{+}+\int_{\partial \Omega}(w-c)^{+} .
$$

And if $a \notin \operatorname{Dom}(\beta)$ (so assuming a is smooth), we take $b^{m}=b+\frac{1}{m} a$, which belongs to $\gamma^{m, k(m)}(a)$ and satisfies

$$
\lim _{m \rightarrow \infty} b^{m}=b .
$$

Now, since $\left[\left(u_{n}\right)_{r}^{m, k},\left(z_{n}\right)_{r}^{m, k},\left(w_{n}\right)_{r}^{m, k}\right]$ is a weak solution of $\left(S_{z, w}^{\gamma^{m, k}, \beta_{r}^{m, k}}\right)$, we have

$$
\int_{\Omega}\left(\left(z_{n}\right)_{r}^{m, k}-b^{m}\right)^{+}+\int_{\partial \Omega}\left(\left(w_{n}\right)_{r}^{m, k(m)}-\beta_{r}^{m, k}(a)\right)^{+} \leqslant \int_{\Omega}\left(z-b^{m}\right)^{+}+\int_{\partial \Omega}\left(w-\beta_{r}^{m, k}(a)\right)^{+} .
$$

Then, letting $r$ go to $+\infty$ and having in mind that $\lim _{r} \beta_{r}^{m, k}(a)=+\infty$, we get

$$
\int_{\Omega}\left(\left(z_{n}\right)^{m, k}-b^{m}\right)^{+} \leqslant \int_{\Omega}\left(z-b^{m}\right)^{+} .
$$

Take the subsequence $k(m)$ used in 48 . Then, taking limits when $m$ goes to $+\infty$ in 60 with $k=k(m)$, we get

$$
\int_{\Omega}\left(z_{n}-b\right)^{+} \leqslant \int_{\Omega}(z-b)^{+} .
$$

Now, letting $n$ go to $+\infty$ in 59 and 61 , we have

$$
\int_{\Omega} z_{b} \leqslant \int_{\Omega}(z-b)^{+}
$$

and therefore $z_{b}=(z-b)^{+}$. Hence, for any $b \geqslant 0$,

$$
\left(z_{n}-b\right)^{+} \rightarrow(z-b)^{+} \quad \text { weakly in } L^{1}(\Omega) .
$$

Similarly, we can get

$$
\left(z_{n}+b\right)^{-} \rightarrow(z+b)^{-} \text {weakly in } L^{1}(\Omega) .
$$

From these convergences we deduce that $z_{n} \rightarrow z$ in $L^{1}(\Omega)$, and the proof is complete. 


\section{Acknowledgements}

We would like to thank to B. Andreianov for many interesting discussions about the uniqueness part of the paper. This work has been performed during the visits of the first, third and fourth authors to the Universite de Picardie Jules Verne and the visit of the second author to the Universitat de València. They thank these institutions for their support and hospitality. The first, third and fourth authors have been partially supported by the Spanish MEC and FEDER, project MTM2005-00620, and by the Generalitat Valenciana, project ACOMP06/196.

\section{REFERENCES}

1. AIKI, T. Multi-dimensional two-phase Stefan problems with nonlinear dynamic boundary conditions. Nonlinear analysis and applications (Warsaw, 1994), GAKUTO Internat. Ser. Math. Sci. Appl. 7, Gakkōtosho, Tokyo (1996), 1-25. Zbl 0868.35061 MR 1422924

2. Alt, H. W., \& Luckhaus, S. Quasilinear elliptic-parabolic differential equations. Math. Z. 183 (1983), 311-341. Zbl 0497.35049 MR 0706391

3. Amann, H., \& FilA, M. A Fujita-type theorem for the Laplace equation with a dynamical boundary condition. Acta Math. Univ. Comenian. (N.S.) 66 (1997), 321-328. Zbl 0922.35053 MR 1620441

4. Ammar, K., \& Wittbold, P. Existence of renormalized solutions of degenerate elliptic-parabolic problems. Proc. Roy. Soc. Edinburgh Sect. A 133 (2003), 477-496. Zbl 1077.35103 MR 1983682

5. Andreianov, B., \& IgBida, N. Revising uniqueness for a nonlinear diffusion-convection equation. J. Differential Equations 227 (2006), 69-79. Zbl pre05044046 MR 2233954

6. Andreu, F., Igbida, N., Mazón, J. M., \& Toledo, J. $L^{1}$ existence and uniqueness results for quasilinear elliptic equations with nonlinear boundary conditions. Ann. Inst. H. Poincaré Anal. Non Linéaire, to appear.

7. Andreu, F., Mazón, J. M., Segura de León, S., \& Toledo, J. Quasi-linear elliptic and parabolic equations in $L^{1}$ with nonlinear boundary conditions. Adv. Math. Sci. Appl. 7 (1997), 183-213. Zbl 0882.35048 MR 1454663

8. Astarita, G., \& Marrucci, G. Principles of Non-Newtonian Fluid Mechanics. McGraw-Hill, New York (1974).

9. Bejenaru, I., Díaz, J. I., \& VRabie, I. I. An abstract approximate controllability result and applications to elliptic and parabolic systems with dynamic boundary conditions. Electron. J. Differential Equations 2001, no. 50, 19 pp. Zbl 0983.93005 MR 1846666

10. BÉnilan, Ph. Équations d'évolution dans un espace de Banach quelconque et applications. Thesis, Univ. Orsay, 1972.

11. Bénilan, Ph., Boccardo, L., Gallouët, Th., Gariepy, R., Pierre, M., \& VÁzquez, J. L. An $L^{1}$-theory of existence and uniqueness of solutions of nonlinear elliptic equations. Ann. Scuola Norm. Sup. Pisa Cl. Sci. (4) 22 (1995), 241-273. Zbl 0866.35037 MR 1354907

12. Bénilan, Ph., Brezis, H., \& Crandall, M. G. A semilinear equation in $L^{1}\left(R^{N}\right)$. Ann. Scuola Norm. Sup. Pisa Cl. Sci. (4) 2 (1975), 523-555. Zbl 0314.35077 MR 0390473

13. BÉnilan, Ph., \& Crandall, M. G. Completely accretive operators. Semigroup Theory and Evolution Equations (Delft, 1989), Lecture Notes in Pure and Appl. Math. 135, Dekker, New York (1991), 41-75. Zbl 0895.47036 MR 1164641

14. BÉnilan, Ph., Crandall, M. G., \& PAzy, A. Evolution Equations Governed by Accretive Operators. Book to appear.

15. Bénilan, Ph., Crandall, M. G., \& Sacks, P. Some $L^{1}$ existence and dependence results for semilinear elliptic equations under nonlinear boundary conditions. Appl. Math. Optim. 17 (1988), 203224. Zbl 0652.35043 MR 0922980 
16. Bénilan, Ph., \& Wittbold, P. On mild and weak solutions of elliptic-parabolic problems. $A d v$. Differential Equations 1 (1996), 1053-1073. Zbl 0858.35064 MR 1409899

17. Bertsch, M., \& Hulshof, J. Regularity results for an elliptic-parabolic free boundary problem. Trans. Amer. Math. Soc. 297 (1986), 337-350. Zbl 0643.35051 MR 0849483

18. Blanchard, D., \& Porretta, A. Stefan problems with nonlinear diffusion and convection. $J$. Differential Equations 210 (2005), 383-428. Zbl 1075.35112 MR 2119989

19. BocCARdo, L., \& GallouËt, T. Nonlinear elliptic equations with right-hand side measures. Comm. Partial Differential Equations 17 (1992), 641-655. Zbl 0812.35043 MR 1163440

20. BrÉZIS, H. Problèmes unilatéraux. J. Math. Pures Appl. (9) 51 (1972), 1-168. Zbl 0237.35001 MR 0428137

21. BREZIS, H. Opérateurs maximaux monotones et semi-groupes de contractions dans les espaces de Hilbert. North-Holland (1973). Zbl 0252.47055 MR 0348562

22. Carrillo, J. Entropy solutions for nonlinear degenerate problems. Arch. Ration. Mech. Anal. 147 (1999), 269-361. Zbl 0935.35056 MR 1709116

23. CARrillo, J., \& Wittbold, P. Uniqueness of renormalized solutions of degenerate elliptic-parabolic problems. J. Differential Equations 156 (1999), 93-121. Z Zbl 0932.35129|

24. Colli, P., \& Rodrigues, J. F. Diffusion through thin layers with high specific heat. Asymptotic Anal. 3 (1990), 249-263. Zbl 0724.35010 MR 1076450

25. CRAndall, M. G. An introduction to evolution governed by accretive operators. Dynamical Systems (Providence, RI, 1974), Vol. I, Academic Press, New York (1976), 131-165. Zbl 0339.35049 MR 0636953

26. CRANK, J. Free and Moving Boundary Problems. North-Holland, Amsterdam (1977).

27. DiBenedetto, E., \& Friedman, A. The ill-posed Hele-Shaw model and the Stefan problem for supercooled water. Trans. Amer. Math. Soc. 282 (1984), 183-204. Zbl 0621.35102 MR 0728709

28. Duvaut, G., \& Lions, J. L. Inequalities in Mechanics and Physics. Springer, Berlin (1976). Zbl 0331.35002 MR 0521262

29. Elliott, C. M., \& JANOVSKÝ, V. A variational inequality approach to Hele-Shaw flow with a moving boundary. Proc. Roy. Soc. Edinburgh Sect. A 88 (1981), 93-107. Zbl 0455.76043 MR 0611303

30. Escher, J. Quasilinear parabolic systems with dynamical boundary conditions. Comm. Partial Differential Equations 18 (1993), 1309-1364. Zbl 0816.35059 MR 1233197

31. GrobbelaAR-VAN DALSEN, M. On $B$-evolution theory and dynamic boundary conditions on a portion of the boundary. Appl. Anal. 40 (1991), 151-172. Zbl 0735.35070 MR 1095411

32. Hintermann, T. Evolution equations with dynamic boundary conditions. Proc. Roy. Soc. Edinburgh Sect. A 113 (1989), 43-60. Zbl 0699.35045 MR 1025453

33. Hulshof, J. Bounded weak solutions of an elliptic-parabolic Neumann problem. Trans. Amer. Math. Soc. 303 (1987), 211-227. Zbl 0668.35048 MR 0896018

34. IgBida, N. The mesa-limit of the porous-medium equation and the Hele-Shaw problem. Differential Integral Equations 15 (2002), 129-146. Zbl 1011.35080 MR 1870466

35. IGBidA, N. Hele-Shaw type problems with dynamical boundary conditions. Submitted.

36. Igbida, N. From fast to very fast diffusion in the nonlinear heat equation. Trans. Amer. Math. Soc., to appear.

37. Igbida, N. A nonlinear diffusion problem with localized large diffusion. Comm. Partial Differential Equations 29 (2004), 647-670. Zbl 1065.35137 MR 2059144

38. Igbida, N., \& KirAne, M. A degenerate diffusion problem with dynamical boundary conditions. Math. Ann. 323 (2002), 377-396. Zbl 1001.35072 MR 1913047

39. Igbida, N., \& Urbano, J. M. Uniqueness for nonlinear degenerate problems. NoDEA Nonlinear Differential Equations Appl. 10 (2003), 287-307. Zbl 1024.35054 MR 1994812 
40. Kenmochi, N. Neumann problems for a class of nonlinear degenerate parabolic equations. Differential Integral Equations 3 (1990), 253-273. Zbl 0724.35056 MR 1025177

41. Kinderlehrer, D., \& Stampacchia, G. An Introduction to Variational Inequalities and Their Applications. Pure Appl. Math. 88, Academic Press, New York (1980). Zbl 0457.35001 MR 0567696

42. Kondic, L., Palffy-Muhoray, P., \& Shelley, M. J. Models of non-Newtonian Hele-Shaw flow. Phys. Rev. E 54 (1996), 4536-4539.

43. Kondic, L., Shelley, M. J., \& Palffy-Muhoray, P. Non-Newtonian Hele-Shaw flow and the Saffman-Taylor instability. Phys. Rev. Lett. 80 (1998), 1433-1437.

44. Langer, R. E. A problem in diffusion or in the flow of heat for a solid in contact with fluid. Tôhoku Math. J. 35 (1932), 260-275. Zbl 0005.09604

45. Lieberman, G. M. Boundary regularity for solutions of degenerate elliptic equations. Nonlinear Anal. 12 (1988), 1203-1219. Zbl 0675.35042 MR 0969499

46. Lions, J. L. Quelques méthodes de résolution des problèmes aux limites non linéaires. Dunod, Paris (1969). Zbl 0189.40603 MR 0259693

47. Primicerio, M., \& Rodrigues, J. F. The Hele-Shaw problem with nonlocal injection condition. Nonlinear Mathematical Problems in Industry, II (Iwaki, 1992), GAKUTO Internat. Ser. Math. Sci. Appl. 2, Gakkōtosho, Tokyo (1993), 375-390. Zbl 0875.35157| MR 1370478

48. Rodrigues, J. F. Variational methods in the Stefan problem. Phase Transitions and Hysteresis (Montecatini Terme, 1993), Lecture Notes in Math. 1584, Springer, Berlin (1994), 147-212. Zbl 0819.35154 MR 1321833

49. Rodrigues, J. F., Solonnikov, V. A., \& Yi, F. On a parabolic system with time derivative in the boundary conditions and related free boundary problems. Math. Ann. 315 (1999), 61-95. Zbl 0940.35211 MR 1717544

50. SU, N. Multidimensional degenerate diffusion problem with evolutionary boundary condition: existence, uniqueness, and approximation. Flow in Porous Media (Oberwolfach, 1992), Internat. Ser. Numer. Math. 114, Birkhäuser, Basel (1993), 165-178. Zbl 0805.76075 MR 1276502

51. van Duyn, C. J., \& Peletier, L. A. Nonstationary filtration in partially saturated porous media. Arch. Ration. Mech. Anal. 78 (1982), 173-198. Zbl 0502.76101 MR 0648943

52. Vrabie, I. I. Compactness Methods for Nonlinear Evolutions. Pitman Monogr. Surveys Pure Appl. Math. 75, Longman, Harlow (1995). Zbl 0842.47040 MR 1375237

53. Ziemer, W. P. Weakly Differentiable Functions. Grad. Texts in Math. 120, Springer, New York (1989). Zbl 0692.46022 MR 1014685 